\title{
Solid-state nanopore systems: from materials to applications
}

\author{
Yuhui $\mathrm{He}^{1}$, Makusu Tsutsui $\mathbb{C}^{2}$, Yue Zhou ${ }^{1}$ and Xiang-Shui Miao (D)
}

\begin{abstract}
Ion transport and hydrodynamic flow through nanometer-sized channels (nanopores) have been increasingly studied owing to not only the fundamental interest in the abundance of novel phenomena that has been observed but also their promising application in innovative nanodevices, including next-generation sequencers, nanopower generators, and memristive synapses. We first review various kinds of materials and the associated state-of-the-art processes developed for fabricating nanoscale pores, including the emerging structures of DNA origami and 2-dimensional nanopores. Then, the unique transport phenomena are examined wherein the surface properties of wall materials play predominant roles in inducing intriguing characteristics, such as ion selectivity and reverse electrodialysis. Finally, we highlight recent progress in the potential application of nanopores, ranging from their use in biosensors to nanoporebased artificial synapses.
\end{abstract}

\section{Introduction}

Fluid mechanics is the study of fluid transport in a macroscopic conduit and is relevant to many disciplines in engineering and biology. On the other hand, the past decades have witnessed a tremendous surge in exploring novel transport phenomena in microscopic channels called nanopores, whose critical dimensions are from $1 \mathrm{~nm}$ to $100 \mathrm{~nm}^{1-3}$. Originally, such nanoscale systems were found in membranes of biological structures such as organelles, cells, and organs that play vital roles in regulating the transport of ions and molecules for not only maintaining physiological conditions but also transducing signals to realize various physical tasks ${ }^{4}$. Recent advances in semiconductor technologies have paved the way for addressing and mimicking the intriguing mechanisms of infinitesimal holes by allowing the fabrication of nanoscopic synthetic channels. These advances have also spurred progressive efforts toward harnessing the

\footnotetext{
Correspondence: Makusu Tsutsui (tsutsui@sanken.osaka-u.ac.jp) Xiang-Shui Miao (miaoxs@hust.edu.cn)

${ }^{1}$ Wuhan National Laboratory for Optoelectronics, School of Optical and Electronic Information, Huazhong University of Science and Technology, 430074 Wuhan, China

${ }^{2}$ The Institute of Scientific and Industrial Research, Osaka University, 8-1 Mihogaoka, Ibaraki, Osaka 567-0047, Japan
}

ingenious functions of these nanopores for practical applications since compared to their biological counterparts, these artificial nanostructures are more stable and allow for tunable designs.

A unique property of nanopores is that mass transport is now affected significantly by the properties of the solid-liquid interface at the wall surface, as the space is confined to the length scale of forces derived from coulombic, van der Waals, and hydrophobic/hydrophilic interactions. The surface charge effect is a good example commonly found in nanofluidic channels of various material and structural designs, where dense counterions that are electrostatically attracted to the wall become comparable to or even overwhelm the bulk ion concentration $^{5}$. This imbalance between coions and counterions results in an electrically charged solution within the channels, giving rise to anomalous transport behavior such as electrical current polarization and reverse electrodialysis. These novel findings have opened new horizons for attractive applications ranging from sequencing to iontronics.

This review is organized to highlight recent progress in the field of solid-state nanopore technology (Fig. 1). Since it is not possible to cover the entire research frontier of 


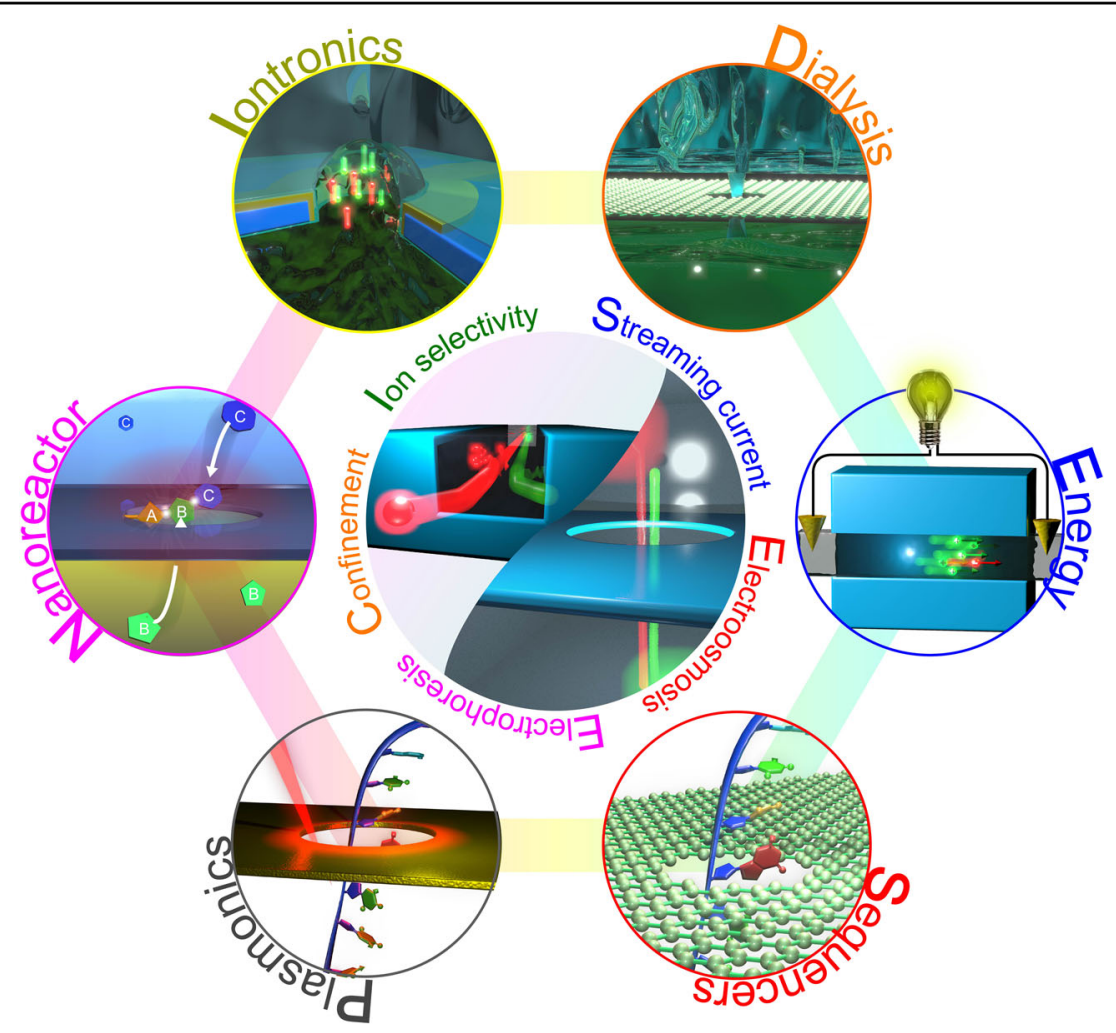

Fig. 1 Fundamental properties and potential applications of solid-state nanopores. The nanoscale hole can be a sensor to detect and analyze single-molecules and -particles. Material and structural designs also enables ion selective membranes useful for the iontronics and energy harvesting applications.

nanofluidics, we recommend reading the recent excellent articles on topics that are not included in the present review $^{6-10}$. We start by introducing fabrication methods developed for creating nanofluidic systems with a variety of materials and in an assortment of shapes. We then describe state-of-the-art studies reporting novel mass transport phenomena in nanoscale conduits with an emphasis on ion transport characteristics. After that, we outline the latest advances achieved in some innovative applications from long-studied single-molecule sequencing to the emerging concept of ionic memristors. Finally, we discuss the perspectives and challenges for the future development of nanofluidics devices.

\section{Materials and fabrication}

Nanofluidics deals with hydrodynamic flows restricted within the nanoscale region in at least one dimension. Devices fabricated with nanometer or subnanometer gaps or channels guiding this extremely tiny fluid are called nanochannels or nanopores. These terms are selectively used to roughly describe length-to-diameter aspect ratio geometries ${ }^{7}$. However, for the sake of clarity, we consider nanoscale conduits as nanopores regardless of the channel architecture since there is practically no clear definition for discriminating them. Below, we introduce the fabrication procedures of solid-state nanopores made of various kinds of materials and resulting in an assortment of shapes.

\section{Silica-based nanopores}

The direct irradiation of focused ions or electron beams can drill a nanopore in a thin membrane made of silicon dioxide $\left(\mathrm{SiO}_{2}\right)$ or silicon nitride $\left(\mathrm{SiN}_{\mathrm{x}}\right)$, as illustrated in Fig. $2 \mathrm{a}^{11,12}$. The substrate is a piece of a silicon wafer. On the wafer, thermally grown $\mathrm{SiO}_{2}$ covers both surfaces. Alternatively, thin layers of $\mathrm{SiN}_{\mathrm{x}}$ are deposited by lowpressure chemical vapor deposition. After forming a window by partially removing the thin film, the exposed $\mathrm{Si}$ surface is deeply etched with $\mathrm{KOH}$. As a result, one obtains a nice flat membrane with a thickness $<50 \mathrm{~nm}$ as long as the residual stress in the thin layer is not significant. When focusing high-intensity ions or electron beams at the membrane, nanopores reaching subnanometer-scale diameters can be formed. By simultaneously monitoring the current on the other side of the membrane, the moment the nanopore opens can be determined, thereby preventing further growth of the pore due to overdosing ${ }^{11}$. TEM allows us to confirm the actual diameter of the channel that is formed, which usually has an hourglass-like motif. The size 


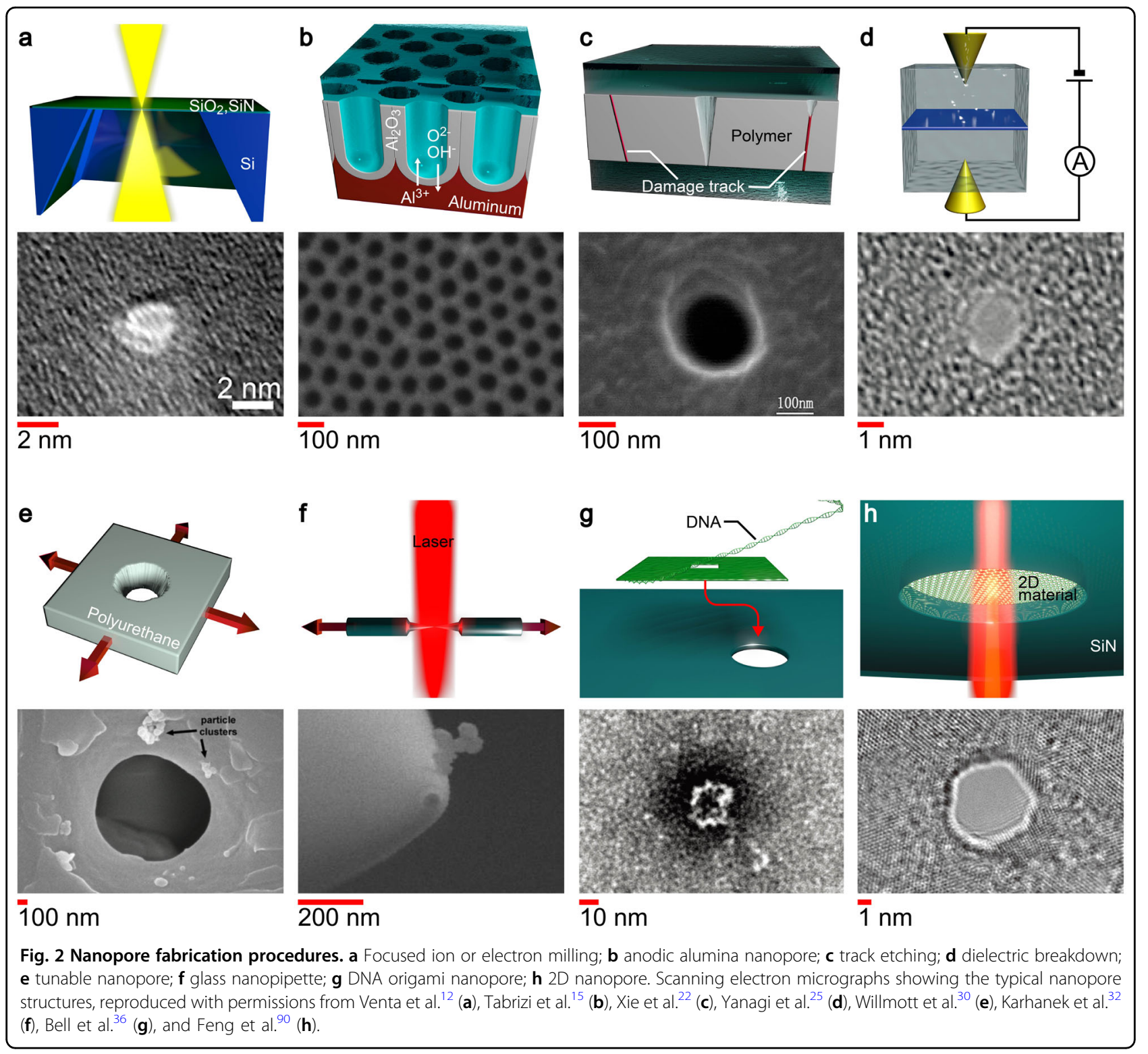

of the nanopore is also adjustable by postirradiation with a low-intensity electron beam ${ }^{13}$. After first being demonstrated for detecting single-molecule DNA by resistive pulse measurements ${ }^{11}$, this fabrication method has been widely employed as a reliable approach to sculpt nanopores with single-nanometer diameters in a solid membrane for the resistive pulse detection of single-molecule proteins and genomes.

\section{Anodic alumina nanopore}

A well-ordered alumina nanopore array can be created by the anodization of an aluminum surface (Fig. 2b). Adding voltage to a mirror-polished aluminum substrate immersed in an acidic solution, such as those made with sulfuric, phosphoric, and oxalic acids, initiates the growth of pores via the electric field-driven dissolution of the liquid-touching $\mathrm{Al}_{2} \mathrm{O}_{3}$ and the concomitant oxidation of the aluminum at the bottom of the hole ${ }^{14,15}$. Due to repulsive interactions between neighboring nanopores, they tend to be self-organized in a densely packed hexagonal arrangement ${ }^{16}$. Here, it is important to meet a condition, which is generally defined by the solution $\mathrm{pH}$ and the current density, to let $\mathrm{Al}^{3+}$ migrate efficiently into the electrolyte solution through the oxide layer. Otherwise, pore formation does not occur, as the $\mathrm{Al}^{3+}$ serves to form an $\mathrm{Al}_{2} \mathrm{O}_{3}$ barrier layer without dissolving in the liquid ${ }^{15}$. Conversely, by carefully adjusting the parameters, one can create uniformly sized nanopores with diameters from $4 \mathrm{~nm}$ to $200 \mathrm{~nm}$ at densities ranging from $10^{12}$ to $10^{15}$ pores $/ \mathrm{m}^{217,18}$. Usually, a two-step process is employed for 
producing anodic alumina nanopores, namely, predefining a pore array pattern on a flat aluminum surface, by nanoimprinting ${ }^{19}$ or preoxidation ${ }^{20}$, before pore formation by anodization. By detaching the membrane or ion milling the closed end of the oxide layer, one can acquire a through-hole nanopore array. A unique advantage of this fabrication procedure is that nanoscale conduits with welldefined shapes can be produced with a simple chemical process, which is less suitable for single-molecule sensor applications but more suitable for producing the platforms of energy harvesters and nanoreactors.

\section{Track-etched nanopore}

Conical-shaped nanopores can be created in a polymer membrane by a method called track etching (Fig. 2c). It uses an isolated polymer thin film often made of polycarbonate or poly(ethylene terephthalate $)^{21,22}$. By irradiating a high-energy heavy ion beam in the range of $\mathrm{MeV}$ to $\mathrm{GeV}$, the polymer chains are locally degraded, thus generating damage tracks that are selectively dissolved by immersing the membrane in an alkaline solution, such as $\mathrm{NaOH}$. As a result, long nanopores penetrate through the polymer. Whereas it is preferable to have only one pore to study the ion transport characteristics, the substrate usually contains multiple damage tracks, making it difficult to drill only a single channel. Special care should therefore be taken to choose and use only one channel among the sporadically formed track-etched holes under an optical microscope $\mathrm{e}^{21}$. It has also been reported that one can open only one pore by simultaneously recording the temporal change in the cross-membrane ionic current during wet etching ${ }^{23}$. The tips of the holes can be made as small as $10 \mathrm{~nm}$, while having a large, $\mu \mathrm{m}$-scale opening on the other side. The overall shape can be controlled by applying voltage during etching, which serves to enlarge the opening and blunt the conical motif ${ }^{21}$. This mechanism is of particular importance in view of resistive pulse sensing, as a long and narrow conduit connected to the nanopore tip will add nonnegligible ionic resistance, thereby decreasing sensor sensitivity. Due to being a relatively inexpensive process, this method has been extensively used to study fundamental ion transport characteristics in nanofluidic channels.

\section{Dielectric breakdown}

The insulating properties of thin dielectrics are known to be impaired when a critically large electric field is imposed. This phenomenon is called dielectric breakdown and has been found to be a simple method to drill a nanopore in a solid membrane (Fig. 2d) ${ }^{24-26}$. The setup consists of a $\mathrm{SiN}_{\mathrm{x}}$ membrane suspended on a $\mathrm{Si}$ wafer. The top and bottom sides are filled with electrolyte buffer, and voltage is applied across the thin dielectric layer by using two electrodes via a conductive ionic solution.
When measuring the current, it tends to increase gradually with an increasing voltage even before a hole opens due to the leakage current through the thin $\mathrm{SiN}_{\mathrm{x}}$ membrane. Moreover, a current jump is observed by further enlarging the bias that signifies the creation of a nanopore. The size of the pore can be roughly controlled by the voltage and can reach the subnanometer scale. A prerequisite for this method is to use an ultrathin membrane that is $<10 \mathrm{~nm}$ thick to ensure a sufficiently large electric field strength is produced to trigger breakdown. Alternatively, it has been observed that a thin solid film that remains at one end of the hole cannot be ruptured by voltage bias when using a relatively thick membrane, although making the electrolyte solution more alkaline is also reported to help remove this capping layer ${ }^{27}$. Recently, this procedure has been extended to form multiple nanopore arrays by using an atomic force microscopy tip as a movable electrode to pinpoint the location to apply the voltage ${ }^{28}$. Although fine control of channel shape and size remains a challenge, the procedure is simple, requiring only voltage stress to form single nanometer-sized pores; thus, this is a promising process for the mass production of nanopore sensors.

\section{Tunable nanopore (q-Nano)}

Tunable nanopores are commercialized solid-state nanopores $^{29,30}$. The apparatus comprises a sample stage with pullers to mechanically deform an elastic membrane made of thermoplastic polyurethane (Fig. 2e). In a $200-\mu \mathrm{m}$ thick polymer, a conical-shaped hole is formed by drilling with a sharpened tungsten tip. Stretching of the membrane can enlarge the pore to the desired size within the maximum strain loadable to the polymer without causing failure. A detailed protocol has been developed to calibrate the sensor wherein it measures standard synthetic beads to first evaluate the actual pore shape and size. The device has proven useful in detecting particles over a vast size range (from $35 \mathrm{~nm}$ up to $1 \mu \mathrm{m}$ ) by choosing a suitable aperture.

\section{Glass nanopipette}

Glass is a hard and brittle transparent substance widely used as pipettes in laboratories due in part to its excellent chemical inertness. The material is generally made soft and deformable by heating above the glass transition temperature and then deforming the material into the desired shape. This glass sculpture technique allows the formation of a pipette with a nanoscale tip opening $(\text { Fig. 2f })^{31,32}$. The starting structure is a simple glass pipe. A laser beam is then used to irradiate the middle part of the pipe to locally heat and soften that portion. Moreover, a tensile force is applied to gently narrow the softened glass down to several tens of nanometers. This ingenious process is automated by a commercialized laser puller device that can control the parameters, such as the pulling speed, 
to reproducibly fabricate nanopores of a certain size $\mathrm{s}^{33}$. Similar to track-etched nanopores, glass nanopipettes can be obtained without nanofabrication technologies, which has led to its wide application in laboratories to investigate fluid and ion transport in nanofluidic channels.

\section{DNA origami nanopore}

While top-down approaches are useful for sculpting micro- to nanoscale conduits in dielectric substrates, their precision is far from competitive with that of chemistry, as we see in the atomically precise structure of bionanopores. Therefore, intensive efforts have also been devoted to building nanopores by synthesis. In this context, DNA nanotechnology has proven useful to design pores at the nanoscale that use the selectivity of the four nucleobases to freely design nanostructures by assembling molecular building blocks called scaffolds and staples ${ }^{34}$. This bottomup technology, which is called DNA origami, provides a way to form nanopores of well-defined size and shape at a level of precision beyond semiconductor technologies (Fig. 2g) ${ }^{35}$. However, since the molecular assembly approach is not feasible for constructing large structures such as a micrometer-scale membrane, the nanoporeholding DNA structure is made to be relatively small (only several tens of nanometers) ${ }^{36,37}$. Therefore, this structure requires an additional process before being employed as a sensor. Electrophoresis has been found to be a promising strategy that implements the trapping of the negatively charged DNA structure on a solid-state nanopore via strong electrophoretic force under an applied crossmembrane voltage. The captured of the DNA structure on the nanopore can be confirmed by monitoring the temporal change in ionic current. Glass nanocapillaries ${ }^{37}$ and $\mathrm{SiN}_{\mathrm{x}}$ nanopores ${ }^{36}$ have proven useful for suspending a DNA nanopore. It is also possible for the DNA structure to be embedded in a lipid bilayer by attaching a hydrophobic belt to partially cover the hydrophilic phosphate groups in the DNA components ${ }^{38}$. The amazing precision of forming a single nanometer-scale pore together with unique surface properties that are useful for analyte-dependent interactions make DNA origami nanopores a promising platform for single-molecule sequencing.

\section{Nanopores based on 2D materials}

The original idea of nanopores aims to use the Coulter principle for decoding the genome. A prerequisite for single-molecule sequencing by an ionic current is to achieve single- or several-nucleobase resolution in resistive pulse analyses. Accordingly, the thickness of a membrane needs to be comparable to the subnanometer spacing of nucleotides in single-stranded DNA. In this regard, two-dimensional materials have been increasingly studied as promising membranes due to their monoatomic thickness (Fig. 2h) ${ }^{39}$. Three research groups have reported the fabrication and use of a graphene nanopore for detecting polynucleotides ${ }^{40-42}$. They transferred a piece of graphene obtained by the mechanical exfoliation of graphite or the chemical vapor deposition of methane on a metal foil onto a relatively large pore formed in a $\mathrm{SiN}_{\mathrm{x}}$ membrane. A nanopore was then sculpted by focused electron beam milling. While successful in observing single-molecule DNA translocation, the experiments also revealed the hydrophobic nature of graphene, which may ease pore clogging due to the adsorption of the biopolymer on the surface ${ }^{42}$. To overcome this issue, other two-dimensional materials have also been tested, such as boron nitride $(\mathrm{BN})^{43}$, molybdenum disulfide $\left(\mathrm{MoS}_{2}\right)^{44}$, and transition metal carbide ${ }^{45}$. While the materials differ, the transfer and nanopore fabrication processes are basically the same as the pioneering works on graphene. The ultimate thinness of the 2D nanopores prepared with this method makes them an optimal choice for achieving single-nucleobase resolution to realize solid-state nanopore sequencing.

\section{Surface modification and functionalization}

The chemical modification of a wall surface is an effective method for regulating ion transport in a nanopore because of the small volume in the conduit relative to the surrounding surface area ${ }^{46}$. Various functional molecules have been utilized to render novel functionalities. A straightforward example may be $\mathrm{pH}$-tunable $I-V$ characteristics ${ }^{47}$. By adjusting the solution $\mathrm{pH}$ higher or lower than the isoelectric point of functional molecules, the channel wall can be made negatively or positively charged, which can give rise to $\mathrm{pH}$-dependent ionic current rectification behaviors ${ }^{48}$. A polymer brush embedded in a nanopore is also found to demonstrate interesting phenomena, such as temperature-responsive ionic conductance, due to the corresponding changes in the molecular conformation ${ }^{49}$. Beyond regulating ion transport, it also provides a way to control the translocation dynamics of objects inside the channel. Employing molecular probes such as peptides and DNA, particular biomolecules and particles can be temporarily trapped inside the functionalized conduit via specific intermolecular interactions, thereby enabling the bioselective detection of analytes ${ }^{50-52}$. Although the procedure varies depending on the channel material, functionalization is often implemented by the covalent binding of molecules on the wall surface in a solvent. In this regard, metal coatings, such as gold and platinum, are sometimes implemented to make use of thiol groups to strongly anchor the molecules ${ }^{53}$.

\section{Transport phenomena and mechanisms}

Unlike in macroscopic channels, ion, and fluid flow in nanopore systems are affected by surface properties. 
This section summarizes some key phenomena relevant to their potential use in sensor and device applications.

\section{Fundamental ion transport characteristics}

Nanopore devices basically consist of two electrolyte solution-filled chambers separated by a thin dielectric membrane. Applying voltage, the cations and anions are field-driven to move toward the anode and cathode, respectively, which produces a constant ionic current via electrochemical reactions at the electrode-liquid interfaces. Physically, they are diffusive systems despite the nanoscale space allowed for transport due to the ultrashort mean free path of ions in liquid. The ionic conductance can thus be described by Maxwell's model with the constant resistivity of a bulk salt solution $\rho$ as a summation of the resistance inside $\left(R_{\text {pore }}\right)$ and outside $\left(R_{\text {acc }}\right)$ the pores (Fig. 3a, $\left.\mathrm{b}\right)$. Here, whereas $R_{\text {pore }}$ is merely the resistance of an ohmic resistor, for instance, $R_{\text {pore }}=4 \rho L / \pi d^{2}$ in the case of a cylindrical conduit of length $L$ and diameter $d$, the latter is an intriguing factor called the access resistance, which is estimated as ${ }^{54}$ :

$$
R_{\mathrm{acc}}=\frac{1}{2 d n_{0}\left(\mu_{\mathrm{K}}+\mu_{\mathrm{Cl}}\right) e}
$$

while that of the cylindrical nanopore is ${ }^{55}$ :

$$
R_{\mathrm{n}}=\frac{4 L}{\pi d^{2} n_{0}\left(\mu_{\mathrm{K}}+\mu_{\mathrm{Cl}}\right) e}\left(1+\frac{2 l_{\mathrm{Du}}}{R}\right)^{-1}
$$

In the above, $\mu_{\mathrm{K} / \mathrm{Cl}}$ is the electrophoretic mobility of $\mathrm{K}^{+} / \mathrm{Cl}^{-}, n_{0}$ is the imposed concentration of $\mathrm{KCl}$, and $l_{\mathrm{Du}}$ is the Dukhin length defined as the ratio of nanopore surface conductivity over the bulk surface conductivity $l_{\mathrm{Du}}=\kappa_{\mathrm{s}} / \kappa_{\mathrm{b}}$. This simple analytical model is able to explain the ionic current characteristics over a vast range of pore sizes from micro- to nanopores. Recently, the physical origin of ion conductance has also been rigorously explored by all-atom simulations ${ }^{56}$ to shed light on the nontrivial influence of surface charges and the atomistic structure of channels in real experiments (Fig. 3c, d). a

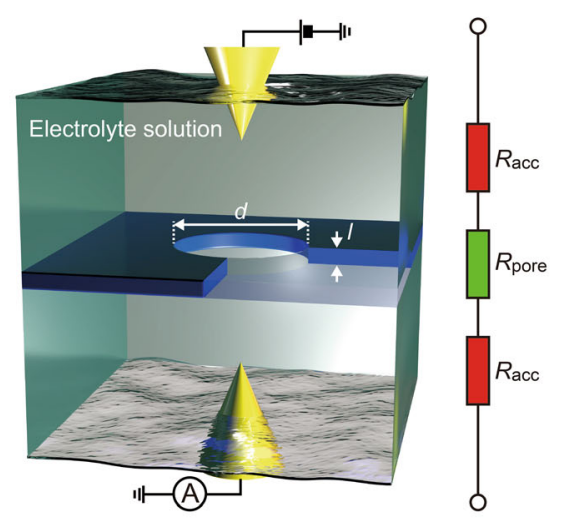

C

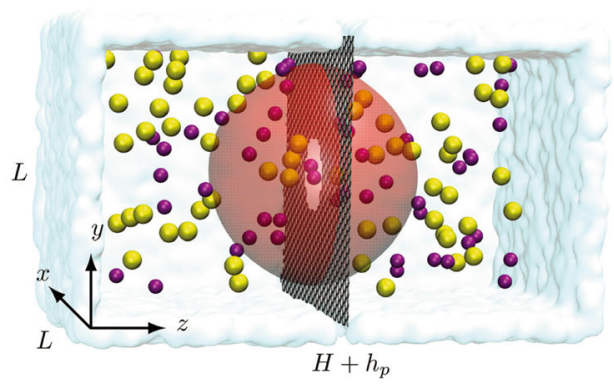

b

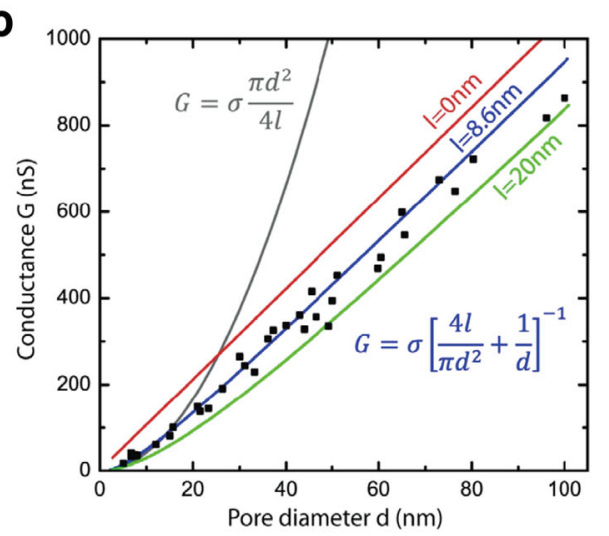

d

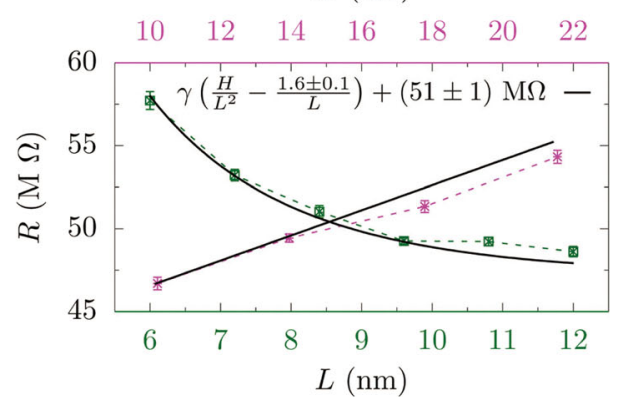

Fig. 3 Maxwell's access resistance. a Equivalent circuit model of a nanopore with diameter $d$ and length / consisting of the resistance $R_{\text {pore }}$ inside the channel connected in series to the access resistance $R_{\text {acc }}$ at the exterior regions. $\mathbf{b}$ Nanopore conductance as a function of the pore diameter. Solid curves denote the theoretical values provided by the analytical model. Reproduced with permission from Kowalczyk et al. ${ }^{57}$. c, $\mathbf{d}$ A model used for estimating the access resistance by all-atom simulations (c) and the obtained cross-pore resistance. Reproduced with permission from Sahu et al. ${ }^{56}$. 
When using low aspect ratio nanopores, the voltage drop in the chambers becomes nonnegligible owing to the increased relative significance of the access resistance over the nanopore resistance: $\Delta V_{\text {acc }} / \mathrm{V} \approx d / L$. It has been verified that only by taking the access resistance into account can the ionic conductance be measured ${ }^{57}$, particularly for ultralow aspect ratio $2 \mathrm{D}$ nanopores ${ }^{40,58}$.

Moreover, the nanopore resistance has been explored experimentally with scanning probe microscopy (SPM) by scanning a tip near nanopore orifices. The corresponding current blockage by the tip apex enables us to map the relative pore resistance increase $\Delta R / R_{0}\left(\Delta R\right.$ and $R_{0}$ are the pore resistance change caused by the tip and the open pore resistance, respectively) as a function of the tip location, nanopore geometry, and salt concentration ${ }^{59}$. The measured $\Delta R / R_{0}$ is found to depend strongly on the imposed salt concentration when small-diameter nanopores are used. The relative resistance rapidly becomes larger at higher $\mathrm{KCl}$ concentrations. This result is attributed to similar mechanisms to those used for explaining the ionic current enhancement observed during DNA translocation under low salt concentration conditions. On the one hand, the volume exclusion effect by the SPM tip near the pore orifice causes an invariant $\Delta R / R_{0}$ with the imposed $\mathrm{KCl}$ concentration. On the other hand, there will be counterions within the EDL surrounding the SPM tip in the solution, which will bring more ions into the conduit (decreasing the resistance, $\Delta R<0$ ). Previous analysis indicates that the smaller the imposed $\mathrm{KCl}$ concentration is, the larger the relative significance of this resistance-reducing effect. In other words, at a high $\mathrm{KCl}$ concentration, the resistance-reducing effect due to the counterions is outperformed by the volume exclusion effect of the charged SPM tips. In this way, a larger $\Delta R / R_{0}$ occurs in the presence of a high salt concentration.

\section{Ion selectivity}

Biological membranes hold channels with unique ion selectivity that admit or reject specific ions, such as $\mathrm{K}^{+}$and $\mathrm{Cl}^{-}$, in response to action potentials. Additionally, it has been demonstrated that ion transport in synthetic nanopores can also be made selective to cations or anions. A typical phenomenon encountered during the currentvoltage measurements of nanofluidic systems is that instead of ohmic behavior, the electrical conductance becomes saturated when $C_{0}$ decreases. Saturation is reported not only in conventional silica nanochannels ${ }^{60,61}$ and polyethylene terephthalate (PET) nanopores ${ }^{62}$, but also in those made from novel materials, such as quasi-1-dimensional boron nitride nanotubes, as seen in Fig. $4 \mathrm{a}-\mathrm{c}^{59}$. This ubiquitous saturation behavior is well explained by considering the electrostatic effect of the surface charges on the channel wall that renders ion selectivity to the synthetic nanochannels ${ }^{60,63}$.

Here, the concept of electrical double layers (EDLs) is crucial to understand ion selectivity. For example, a silica pore wall is charged when in contact with a water solution owing to the hydrolysis reaction $\mathrm{SiOH} \leftrightarrow \mathrm{SiO}^{-}+\mathrm{H}^{+}$. Counterions with opposite types of charges are then electrostatically attracted to the wall surface, while coions of
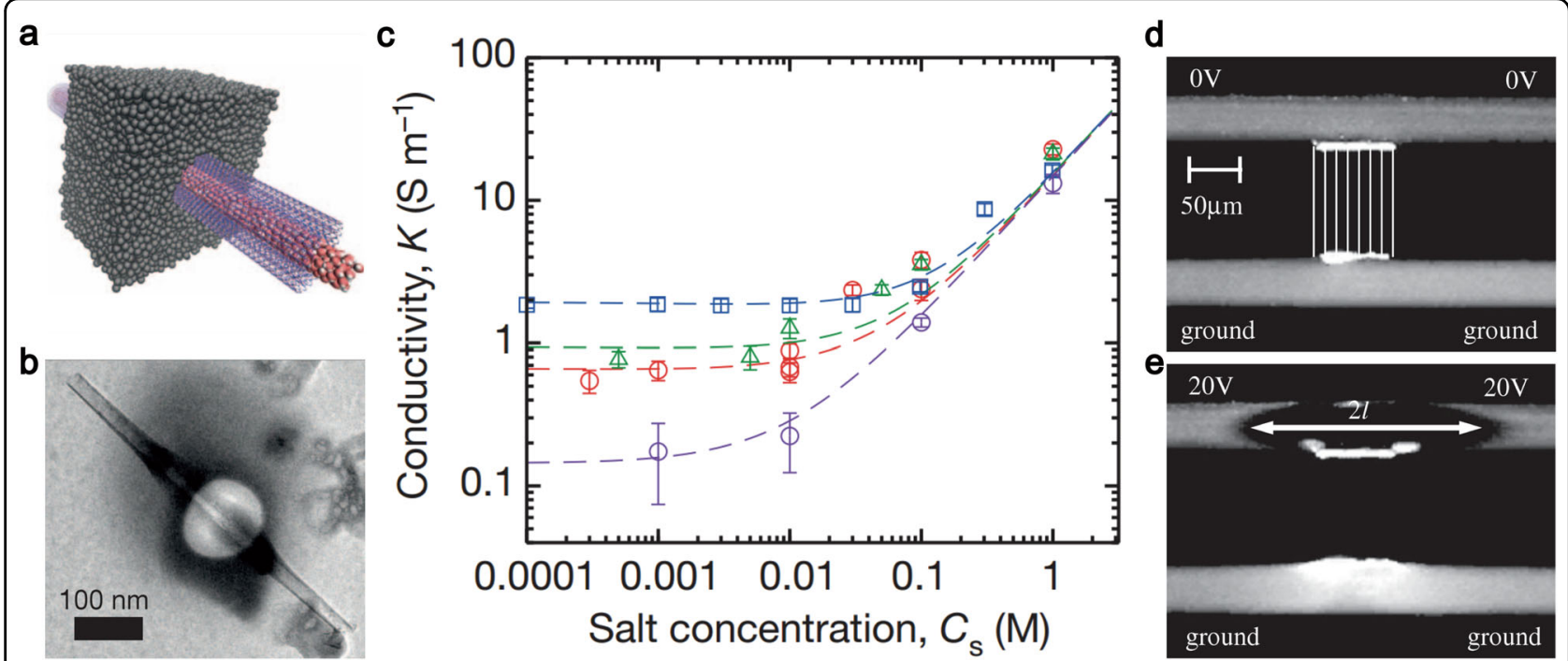

Fig. 4 lon selectivity in solid-state nanopores. a, b Sketch (a) and TEM image (b) of the fabricated transmembrane boron nitride nanotube (BNNT) for nanofluidic measurement. c Measured electrical conductance versus the concentration of imposed $\mathrm{KCl}$ in BNNT systems with various radii and lengths of tubes. $(R, L)=(40 \mathrm{~nm}, 1250 \mathrm{~nm})$ (purple), (29 nm, $900 \mathrm{~nm})$ (red), (22 nm, $1500 \mathrm{~nm})$ (green), and (15 nm, $800 \mathrm{~nm})($ blue) at pH 5. Reproduced with permission from Siria et al. ${ }^{65}$. d, e lon-enrichment and ion-depletion behavior observed with fluorescence in single-gate nanofluidic devices. Reproduced with permission from Kim et al. ${ }^{67}$. 
the same type are repulsed to the inner side. Theoretically, the Poisson-Boltzmann equations can be employed to describe the distributions of the coions, counterions, and electrical potentials in the nanopore system ${ }^{64}$. These equations indicate that both the electrical potential and ion concentration vary significantly in several nanometers around the channel wall, while being kept roughly invariant near the channel center thereby forming an EDL with a thickness defined by the Debye length $\lambda_{\mathrm{D}}$ at the wall-water interfaces. Generally, there are two extreme cases at the higher and lower limits of imposed salt concentrations. One is that $C_{0}$ is so small that the EDL overlaps around the channel center. Since charge screening is ineffective under such conditions, the surface effect tends to dominate transport in the nanopore. The other situation is that the EDL is quite thin because of the large $C_{0}$. Since the imbalance between counterions and coions in the EDL is trivial compared to the bulk salt concentration, the transport behavior becomes more straightforward with little influence of the surface charges. More specifically, we define ion selectivity to measure the significance of the surface charge effect in nanopores with monovalent ions:

$$
\gamma=\frac{\bar{n}_{+}-\bar{n}_{-}}{\bar{n}_{+}+\bar{n}_{-}}
$$

where $\bar{n}_{ \pm}=2 \int_{0}^{R} n_{ \pm} r d r / R^{2}$ is the averaged cation/anion concentration over the channel cross-section. The absolute quantity of ion selectivity will reach the maximum value of 1 given the strong surface effect, indicating that the channel is occupied completely with either cations or anions. On the other hand, $\gamma$ will be 0 in the presence of a negligible surface effect, i.e., when there are almost equal numbers of cations and anions. By noting the electrical neutrality condition in the channel cross-section and using a first-order approximation $\left(\bar{n}_{+}+\bar{n}_{-}\right) \sim C_{0}$, the ion selectivity is further simplified as follows:

$$
\gamma=\frac{-2 \pi R \sigma_{\mathrm{w}}}{e \pi R^{2}\left(\bar{n}_{+}+\bar{n}_{-}\right)} \propto \frac{\sigma_{\mathrm{w}}}{R C_{0}}
$$

In the above, $\sigma_{\mathrm{w}}$ is the density of surface charge on the nanopore wall, and $C_{0}$ is the bulk concentration of the imposed monovalent salt. The above equations predict a more pronounced ion selectivity occurs in fluid channels with a smaller diameter, denser surface charge, and a lower salt concentration. In fact, it has been reported in experiments that by using nanopores with smaller diameters, the conductance per area keeps increasing with a reduced diameter, while saturation of the electrical current starts to be observed at higher salt concentrations (Fig. 4c) $)^{65,66}$.

Further direct evidence of ion selectivity is the concentration polarization at the two ends of a nanopore observed with fluorescence microscopy technology when imposing a cross-channel voltage ${ }^{67}$. By using permselective Nafion membrane systems, only cations are allowed to pass through the nanopores. Negatively charged green fluorescence protein molecules are observed to accumulate and deplete at the low- and high-voltage ends (Fig. 4d-e), which is expected in cation-selective nanopores. Anions, in contrast, can hardly pass through the channel; thus, they tend to accumulate at the entrance. On the other hand, those few anions that pass through are quickly driven to the anode by the electric field, leading to a depletion of anions around the exit.

The ion selectivity explained above is found to yield an abundance of ion transport properties from diode-like characteristics $^{68,69}$ to negative differential resistance ${ }^{70,71}$. Furthermore, solid-state nanopores also serve as a useful platform for exploring the ingenious mechanism behind selective ion and mass transport in biological channels. Recently, Lin et al. $^{72}$ demonstrated calcium gating in molecule-coated nanopores. They functionalized the $\mathrm{SiN}_{\mathrm{x}}$ pore wall surface with triethoxysilylpropylmaleamic acid, which has carboxyl groups to bind $\mathrm{Ca}^{2+}$. The open pore conductance was observed to decrease with an increase in the molar concentration of $\mathrm{CaCl}_{2}$ in a $\mathrm{KCl}$ solution, which was ascribed to the channels shrinking via calcium binding on the membrane surface. This study offers not only unique insights into the physical characteristics of calcium channels in biological systems but also a clue to mimic these functions with solid-state nanopores.

\section{Electroosmotic flow}

One of the aspects that clearly discriminates the ionic characteristics of nanopores from solid-state electron transport counterparts is the intimate role of fluid flow. In this regard, electroosmosis in nanofluidic channels has been intensively studied, which stems from the fact that the electrical double layer moves under an external electrical field due to the net charges inside generating an electrical body force $\vec{f}_{e}$, as seen in the following NavierStokes equation:

$$
\left\{\begin{array}{c}
\rho\left(\frac{\partial \vec{u}}{\partial t}+\vec{u} \cdot \nabla \vec{u}\right)=-\nabla p+\eta \nabla^{2} \vec{u}+\vec{f}_{e} \\
\nabla \cdot \vec{u}=0
\end{array}\right.
$$

where $\vec{f}_{e}$ is given as:

$$
\vec{f}_{e}=\vec{E} \rho_{\mathrm{e}}=e \vec{E} \sum_{i} z_{\mathrm{i}} n_{\mathrm{i}}
$$

In the above, $\vec{u}$ is the velocity field of the fluid, $\rho$ is the fluid density, $p$ is the hydrodynamic pressure, $\eta$ is the fluid viscosity, $\vec{E}$ is the electric field, $z_{\mathrm{i}}$ and $\underline{n}_{\mathrm{i}}$ are the valence and concentration of the ith type of ion, respectively, and $\rho_{\mathrm{e}}$ is the net charge density. As a result, a fluid tends to be moved along the force, which is called electroosmotic flow 
(EOF). Although electroosmosis is a well-studied phenomenon, the characteristics are anticipated to change appreciably in nanopores with a sub-Debye length considering the prominent effects of surface charge. Experimentally, two strategies have been developed to measure EOF velocity. One was an image tracing method where the EOF is evaluated by imposing fluorescent molecules, such as rhodamine $B$, into a nanofluidic system and then measuring their translocation time with a camera or photodetector. The average electroosmotic mobility of borosilicate glass nanopores has been found to be $35 \%$ lower than that in micrometer-scale channels by using this technique ${ }^{73}$. This result is attributed to the prominent viscous force from the channel wall surface now taking a larger part in determining the EOF profile since the EOF overlaps within nanopores with smaller diameters. Nevertheless, several nontrivial side effects have to be carefully assessed when using this image tracing approach. First, it is invasive technology since the electric field within the channel is modulated by the charged fluorescent molecules that are used. Moreover, the electrophoretic and diffusion motion has to be excluded from the measured fluorescence molecule speed since the molecules are added into one chamber and then moved through the nanopore both electrophoretically and diffusively in addition to the advection caused by EOF. Another approach is to estimate the electrical current variation slope upon replacing the more conductive electrolyte solution with a less conductive solution in the channel by EOF. By using this technique, it has been demonstrated that the EOF velocity in small-diameter polymer nanopores is proportional to the applied electric field but is smaller than that in microchannels under the same applied electric field ${ }^{74}$.

The abundance of EOF behaviors is further demonstrated through ionic current modulation $\Delta I$ as well as changes in the velocity of particles and molecules through a nanopore at various salt concentrations ${ }^{61}$. Depending on the $\mathrm{KCl}$ concentration, DNA translocation decreases (Fig. 5a) or increases (Fig. 5b) in an ionic current with some variation. The average translocation speed of DNA is also slower in a $150-\mathrm{mM} \mathrm{KCl}$ solution than in a $500-\mathrm{mM} \mathrm{KCl}$ solution. The above experimental observations suggest that the EOF has a strong dependence on the imposed salt concentration, leading to a varying DNA translocation speed $u_{\mathrm{DNA}}$. Theoretical modeling and calculation can quantitatively explain the above phenomena as being caused by the competing effects of the volume exclusion effect and the anionic DNA-induced EOF effect for ionic current enhancement,

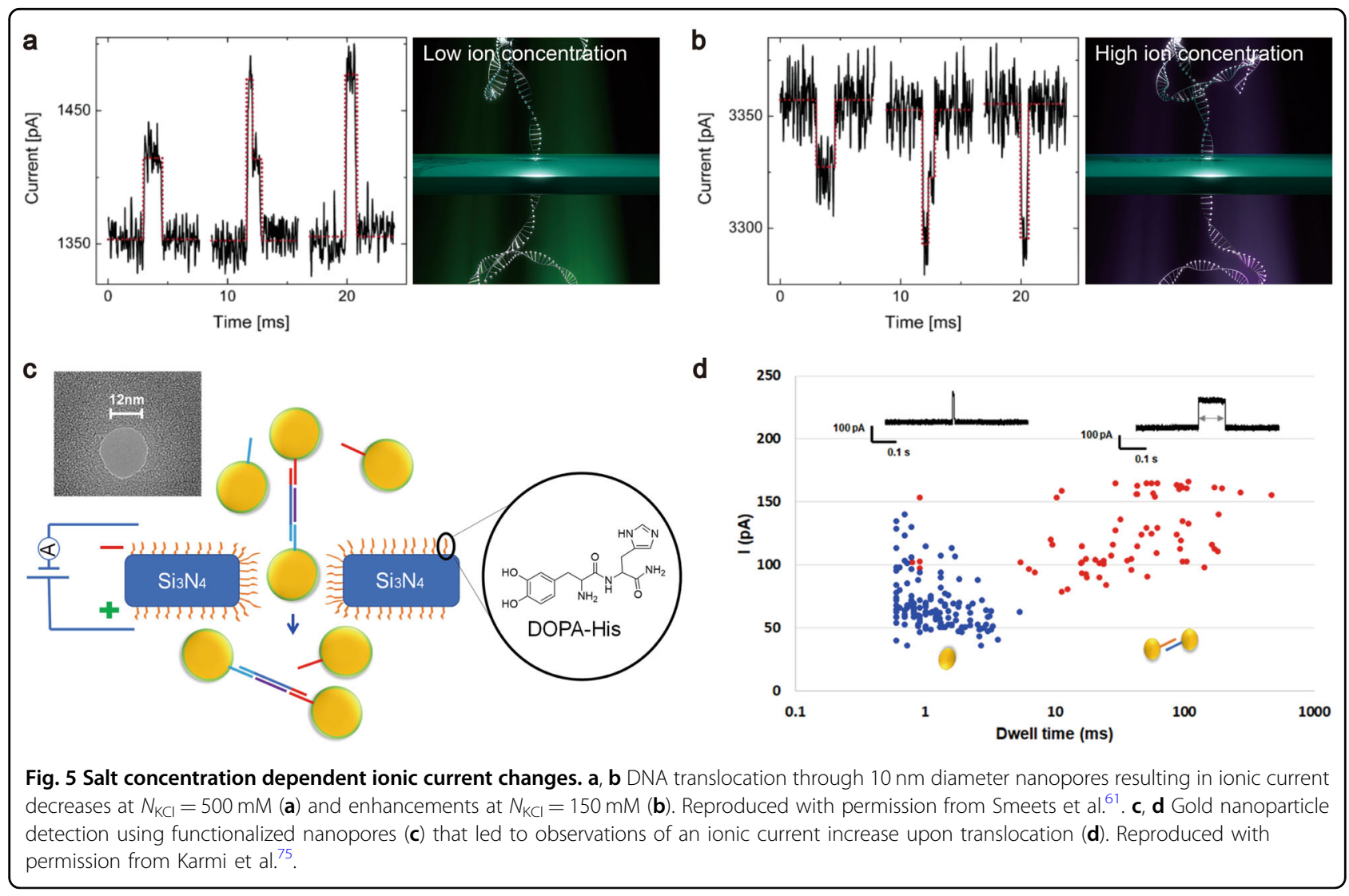



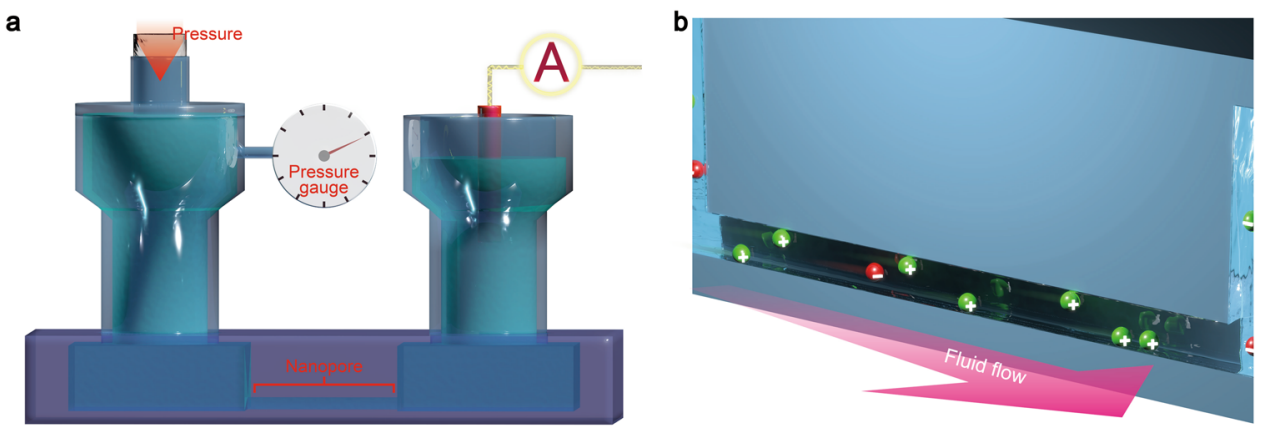

Fig. 6 Streaming current measurements. a Setup for streaming current measurements. External pressure is added to induce fluid flow in a nanopore. The change in the ionic current through the nanopore is recorded as a function of the applied pressure. $\mathbf{b}$ Physical image of the pressure-driven streaming current in the nanopore. The directional flow of the counterions (cations in the image) generates a measurable amount of ionic current.

while the DNA velocity modulation is attributed to the different magnitudes of the $\mathrm{EOF}$ at different $\mathrm{KCl}$ concentrations ${ }^{64}$. Later, this phenomenon is confirmed to be a rather universal phenomenon observed for analytes other than polynucleotides, such as $\mathrm{Au}$ nanoparticles ${ }^{75}$ (Fig. 5c, d) and proteins ${ }^{76}$.

\section{Streaming conductance}

Under mechanical or electrical driving forces, there is both an ionic current $I$ and fluid flow $Q$ in nanopores, which are defined by the following formulas ${ }^{77}$ :

$$
\begin{aligned}
& I=\frac{\mathrm{d} I}{\mathrm{~d} \Delta p} \Delta p+\frac{\mathrm{d} I}{\mathrm{~d} U} \Delta U=S_{\mathrm{str}} \Delta p+\frac{1}{R} \Delta U \\
& Q=\frac{\mathrm{d} Q}{\mathrm{~d} \Delta p} \Delta p+\frac{\mathrm{d} Q}{\mathrm{~d} U} \Delta U=\frac{1}{Z} \Delta p+S_{\mathrm{str}} \Delta U
\end{aligned}
$$

where $\Delta U$ is the applied voltage, $\Delta p$ is the pressure gradient, and $Q=\int_{0}^{2 \pi} d \varphi \int_{0}^{R} u_{\mathrm{z}} r d r$ is the flow rate defined as the area integration of the axial velocity $u_{\mathrm{z}}$ of the fluid over the cross-section of the nanopore. In the above definition, $R$ is the electrical resistance of the pore, $Z$ is the fluidic impedance, and $S_{\text {str }}$ is the so-called streaming conductance. The Onsager relation indicates that the following:

$$
S_{\mathrm{str}}=\frac{\mathrm{d} I}{\mathrm{~d} \Delta p}=\frac{\mathrm{d} Q}{\mathrm{~d} U}
$$

Therefore, experimentally, the streaming conductance can be obtained either by measuring the electrical current upon imposing a hydrodynamic pressure or by measuring the flow rate under an applied voltage. The former denotes a process of mechanical to electrical energy conversion, while the latter does the opposite. Here, we focus on the former mechanism since the pressureinduced electrical potential/current is the process for electrical power generation, while the latter voltage-driven EOF has already been discussed in the preceding section.

An example of an experimental setup for measuring the streaming conductance is described in Fig. 6a, where the air pressure is regulated to add pressure to the flow fluid through a conduit (Fig. 6b) while recording the ionic current through the channel. As depicted in Fig. 6b, the streaming current originates from the pressure-driven flow due to unbalanced counterions. At first glance, it seems that the streaming conductance should be approximately invariant with the imposed salt concentration since the electrical current is the product of flow velocity and the amount of cross-sectional net charge, $I \approx u_{z}\left(\bar{n}_{+}-\bar{n}_{-}\right)$. The former is determined by the applied mechanical pressure, while the latter is provided by the density of the channel wall surface charge. Interestingly, whereas neither factor is related to the salt concentration, the streaming conductance is reported to decrease rapidly at higher $\mathrm{KCl}$ concentrations, while it remains roughly constant when the $\mathrm{KCl}$ concentration is below a critical level ${ }^{63}$. For example, the experimentally measured $S_{\text {str }}$ in a $140-\mathrm{nm}$-high nanopore at $17 \mu \mathrm{M} \mathrm{KCl}$ is three times larger than that at $0.33 \mathrm{M}^{63}$.

Here, the physical mechanism is the different distributions of counterions $n_{ \pm}$along the radial direction when using various concentrations of $\mathrm{KCl}$. Due to the nonslip boundary condition, the cross-pore flow velocity $u_{\mathrm{z}}$ is fastest at the radial center of the nanopore but decreases and becomes zero around the channel wall. In a cylindrical nanopore with a nonslip channel wall, the pressuredriven flow is described by the Hagen-Poiseuille equation $u_{\mathrm{z}}(r)=-(\partial p / \partial z)\left(1-r^{2} / R^{2}\right) \quad R^{4} / 4 \eta$, where $u_{\mathrm{z}}(r)$ is the channel axial component of the flow velocity along the channel radial direction, $\partial p / \partial z$ is the pressure gradient along the channel axial direction, $\eta$ is the viscosity of the solution and $R$ is the channel radius. The profile of $u_{\mathrm{z}}(r)$ is parabola-like. Then, when more net charge is located near the radial center of the channel, the voltage-driven 

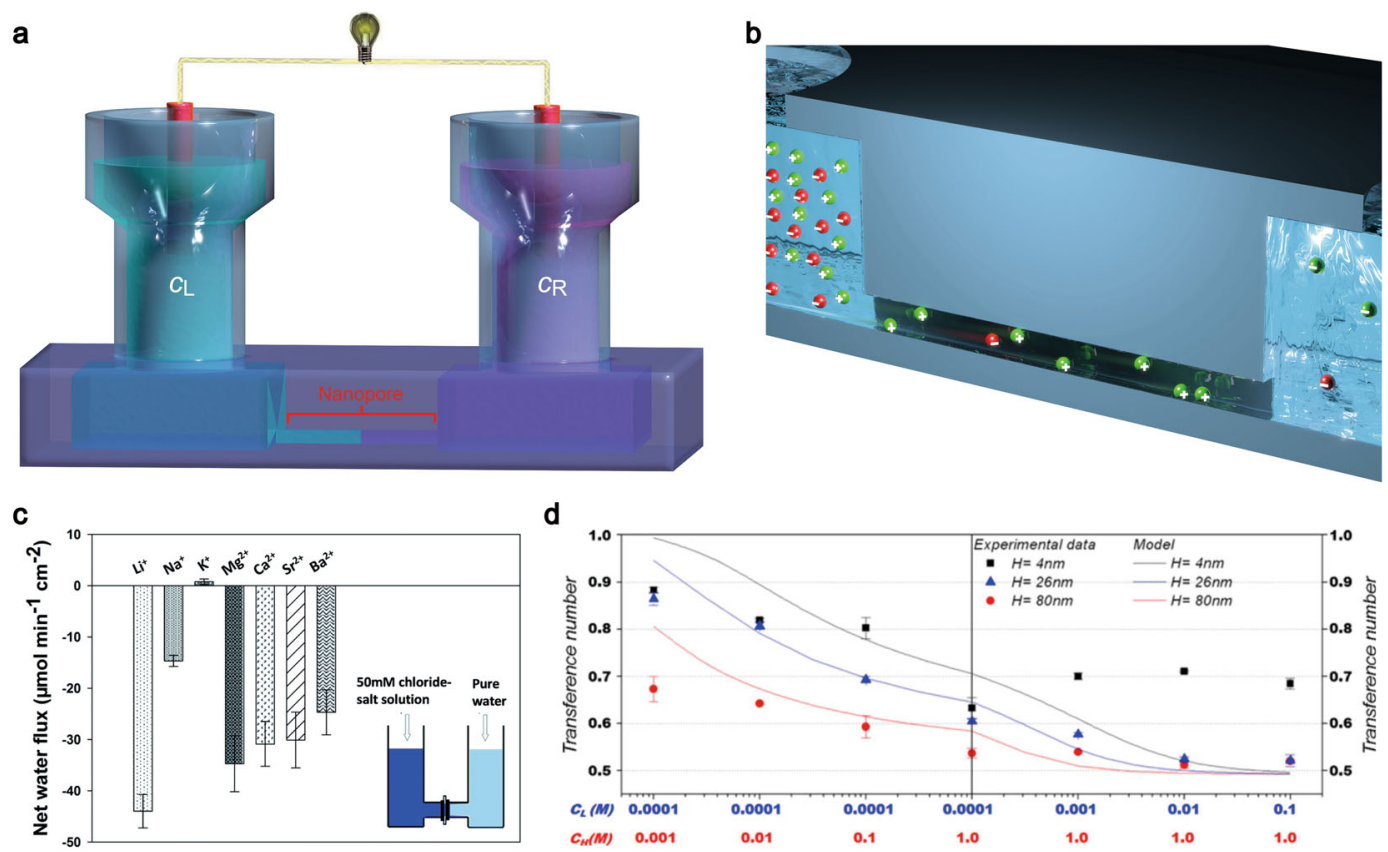

d

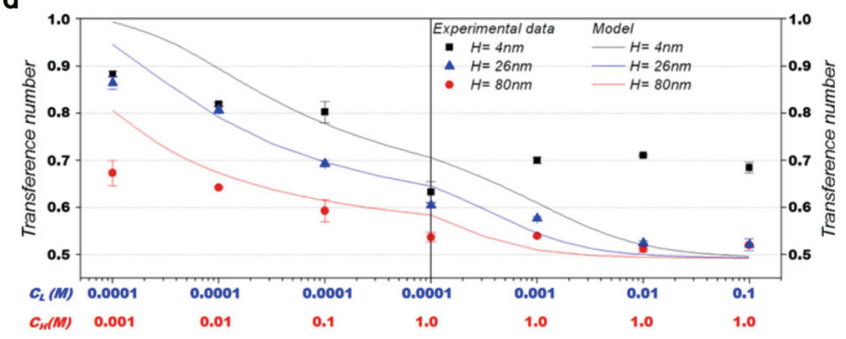

Fig. 7 Reverse electrodialysis in synthetic nanochannels. a System setup for reverse dialysis. Two chambers connected with an ion-selective nanopore were each filled with electrolyte solutions with different ion concentrations. b Schematic model depicting counterion transport from highto low-concentration sides across the nanopore. c Measured net flux of water transport as a function of different electrolyte types. The negative values of $\mathrm{Li}^{+}, \mathrm{Mg}^{2+}, \mathrm{Ca}^{2+}, \mathrm{Sr}^{2+}$, and $\mathrm{Ba}^{2+}$ indicated that the direction of the net flux of water was from the chloride salt solution toward pure water, while the positive direction of $\mathrm{K}^{+}$indicated the opposite. Here, 3.5 (PSS/PAH)-coated $100 \mathrm{~nm}$ membranes were used after standing for $12 \mathrm{~h}$ at $20^{\circ} \mathrm{C}$. Reproduced with permission from Yang et al. ${ }^{81}$. d Open-channel voltage measured in rectangular nanopores with a height $(\mathrm{H})$ of $4 \mathrm{~nm}$ (black-square symbols), $26 \mathrm{~nm}$ (blue-triangle symbols), and $80 \mathrm{~nm}$ (red-round symbols). In the left half, the lower salt concentration $C_{\mathrm{L}}$ was fixed at $0.01 \mathrm{mM}$ at one end while that in the other end $C_{H}$ was tuned from $1 \mathrm{mM}$ to $1 \mathrm{M}$; in the right half, $C_{H}$ was fixed at $1 \mathrm{M}$ while $C_{L}$ was tuned from $0.01 \mathrm{mM}$ to $0.1 \mathrm{M}$. Reproduced with permission from Kim et al. ${ }^{66}$.

electrical current will increase since that is where the flow velocity is largest. Therefore, given roughly the same amount of net charge in the channel cross-section, a smaller salt concentration results in a thicker EDL and a larger proportion of counterions near the radial center of the channel, indicating a larger $S_{\text {str }}$ at a lower salt concentration. In this sense, the experimental observations of decreasing the streaming conductance in nanopores at larger salt concentrations by van der Heyden et al. ${ }^{63,77}$ showed compelling evidence for the EDL variation trend in respect to the salt concentration.

\section{Reverse electrodialysis}

It is known that an electrical voltage will be generated due to the chemical potential gap between solutions with different ion concentrations (Fig. 7a). Thus, reverse electrodialysis (RED) has been proposed to harvest thermal energy by stacking layers of cation/anion selective membranes in an alternating manner between diluted and concentrated solutions (Fig. 7b). Since the crucial technique here is the ion selection property of the membranes, cation- or anion-selective nanopores are also utilized to implement RED and have been further developed as nanoscale power generators ${ }^{66,78-80}$. It has been reported that both the direction and magnitude of the measured open-circuit voltage $\Delta V$ and the water flow by the RED effect are tunable by the ion species and densities ${ }^{81}$. For example, in a 100-nm-diameter nanopore coated with 3.5 layers of poly(allylamine hydrochloride) (PAH) and poly (sodium 4-styrenesulfonate) (PSS) with a chloride solution in one end and pure water in the other end, water flow toward the pure water side has been reported when using $\mathrm{Li}^{+}, \mathrm{Mg}^{2+}, \mathrm{Ca}^{2+}, \mathrm{Sr}^{2+}$, and $\mathrm{Ba}^{2+}$. In contrast, water flow in the opposite direction has been detected when using $\mathrm{K}^{+}$, as shown in Fig. 7c. Another interesting phenomenon is that the induced open-circuit electrical potential $\Delta V$ does not keep increasing when the $\mathrm{KCl}$ concentration difference $C_{\max } / C_{\min }$ increases. Instead, $\Delta V$ reaches a maximum value at a moderate value of $C_{\max } /$ $C_{\text {min }}$ and then decreases at a larger concentration ratio, as seen in Fig. $7 d^{66}$. Not only has it posed a challenge to theoretical understanding of the underlying physical mechanisms, but it also has required more complicated design of the energy harvesting device rather than being 
able to straightforwardly use the large concentration ratio of seawater over river water $\sim 600 \mathrm{mM} / 10 \mathrm{mM}$.

Regarding the theoretical understanding of RED mechanisms, one critical issue is to distinguish the channel wall surface charge-induced electrical potential $\Delta V_{\sigma}$ from that caused by the mobility difference between cations and anions $\Delta V_{\mathrm{D}}$. The thickness of the EDL continues to increase from the concentrated end to the dilute end owing to the imposed salt bias across the nanopore. This mechanism indicates an enhanced ion selectivity along the direction according to Eq. 2. The axial variation of ion selectivity in the channel, as characterized by the diffusion of counterions along the axial direction of the channel, leads to an electrical potential difference $\Delta V_{\sigma}$ at the two ends of the nanopore. On the other hand, the different mobilities between cations and anions, such as $\mathrm{Na}^{+}$and $\mathrm{Cl}^{-}$, also trigger a potential bias $\Delta V_{\mathrm{D}}$. Therefore, $\Delta V_{\sigma}$ is a surface effect that is uniquely present in the nanofluid system, while $\Delta V_{\mathrm{D}}$ is a bulk effect that is ubiquitous in both macroscopic and nanoscopic fluids. To quantitatively analyze $\Delta V_{\sigma}$ and $\Delta V_{\mathrm{D}}$ and their dependence on nanopore device parameters, a space-charge model has been developed to provide insights into RED in salt concentration-biased nanofluidic systems ${ }^{78}$. This model illustrates that depending on the material properties of the channel wall and the imposed salt species, the exclusion effect can counteract or synergize with the diffusion effect, thereby causing some of the various phenomena of RED in nanochannels ${ }^{66,82}$.

\section{Potential applications: nanopore sensors and reactors}

The original idea of nanopore sensing was to use solidstate or biological pores as nanoscale analogs of Coulter counters to detect and analyze single molecules in liquids. Here, we review the developments of practical nanopore sensors and devices in various fields.

\section{Nanopore sequencers}

More than two decades ago, nanopores were found to be a useful tool as a molecular counter ${ }^{83}$. By measuring the times of ionic current blockage caused by the volume exclusion of biomolecules during their electrophoretic transit through nanopores, it can be determined how many molecules have passed ${ }^{84}$. Currently, more sophisticated device structures and measurement metrics are designed with the aim of not only counting move-through events but also recognizing molecules based on features extracted from their ionic current signals, similar to computerized tomography scans ${ }^{84-86}$. Alluring applications such as nanopore-based genome sequencing and protein analysis with revolutionarily enhanced speed at low cost have stimulated a large amount of research activity and tremendous progress in this new horizon in recent decades ${ }^{1,2,87}$.
The ionic current blockage approach in bionanopore sensors largely relies on the fact that different types of biomolecules will have different interaction strengths with the nanopore wall. Consequently, different levels of ionic current blockage are obtained and used as fingerprints to identify diverse types of molecules. A typical example is to exploit the varied binding strengths of four types of nucleotides with engineered Mycobacterium smegmatis porin A (MspA) nanopores ${ }^{4}$. As seen in Fig. 8a, singlestranded DNA in a hairpin conformation can be pulled through an MspA nanopore at a driving voltage of $180 \mathrm{mV}$. An ionic current blockage $\Delta I \sim 240 \mathrm{pA}$ with a duration of several milliseconds is measured. Moreover, by using single-stranded 14-base oligonucleotides with different types of homosequences (adenine, thymine, guanine, and cytosine), different amplitudes of ionic current blockage are observed, as seen in Fig. 8b, c. This result is ascribed to the different strengths of the binding interaction of the four types of nucleotides with the MspA protein ${ }^{4}$. The basic principle of nanopore sequencing thus suggests that nanopores should be highly stable, remain chemically functional over a large variation in environmental temperatures and have discriminable binding strengths with different nucleotides ${ }^{1}$. The ionic current blockage approach has now progressed from the laboratory to industry owing to its overwhelming advantages in increasing sequencing speed and cost-effectiveness compared to traditional approaches, such as sequencing by synthesis. A leading company of this new-generation sequencing technology is Oxford Nanopore ${ }^{\circledR}$, which has developed several U-disk-like nanopore sequencing devices called MinION that have already demonstrated a sequencing alignment accuracy as high as $97 \%$ by thirdparty tests ${ }^{88}$.

On the other hand, there has been a continuing development to establish solid-state devices that mimic the single-molecule sensing capability of biological channels for more robust and low-cost sequencers ${ }^{1-3}$. The conventional structure consists of a 50-nm-thick $\mathrm{SiN}_{\mathrm{x}}$ membrane on a Si wafer wherein a single nanometer-sized pore is sculpted by directly irradiating an ion or electron beam ${ }^{11-13}$. The Coulter principle allows us to sense the electrophoretic translocation of single polynucleotides by measuring the cross-membrane ionic current. Since pioneering works in the 2000s, tremendous efforts have been devoted to enhancing the spatial resolution of solid-state nanopore sensing by reducing the length of channels through the use of thinner membranes. Eventually, three groups succeeded in fabricating pores in an atomically thin sheet of carbon, i.e., graphene nanopores ${ }^{40-42}$. Later, other $2 \mathrm{D}$ materials were also tested, such as boron nitrides ${ }^{89}$, transition metal carbides ${ }^{45}$, and $\mathrm{MoS}_{2}{ }^{90}$, to improve their notoriously hydrophobic characteristics ${ }^{91}$. Among them, $\mathrm{MoS}_{2}$ has been found to be a promising candidate for realizing 

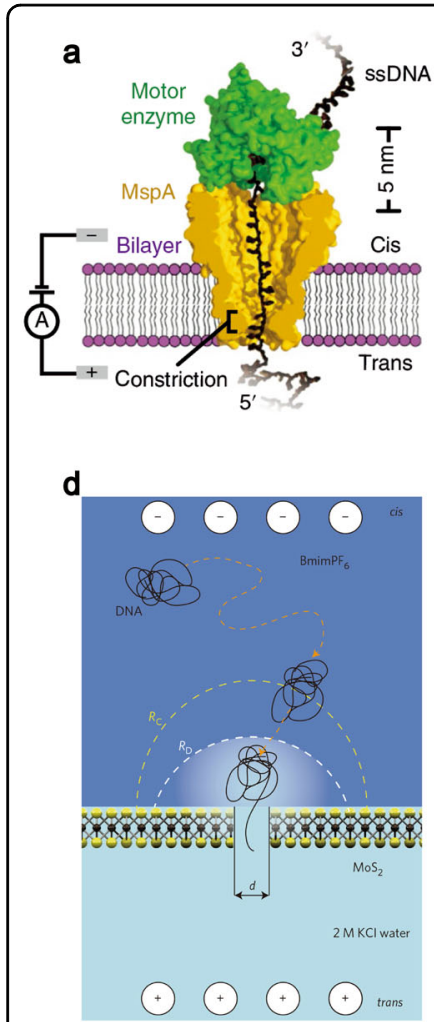

b

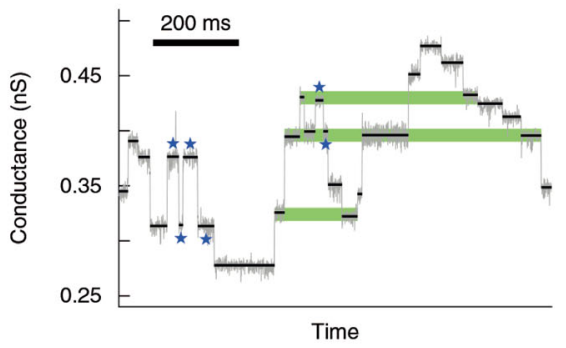

$\mathbf{e}$
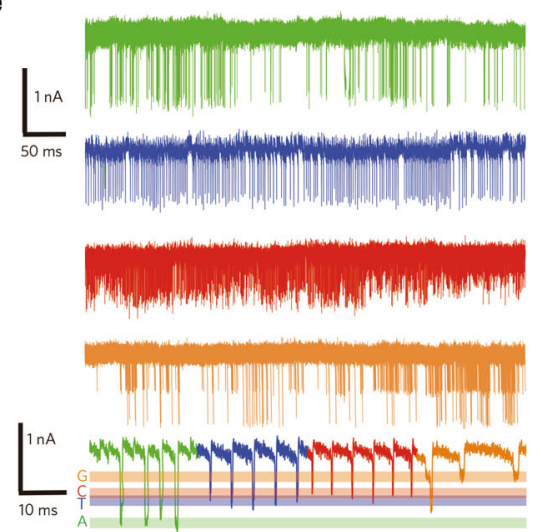

C

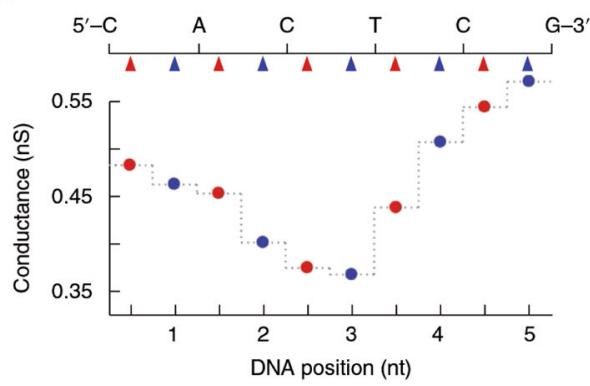

f

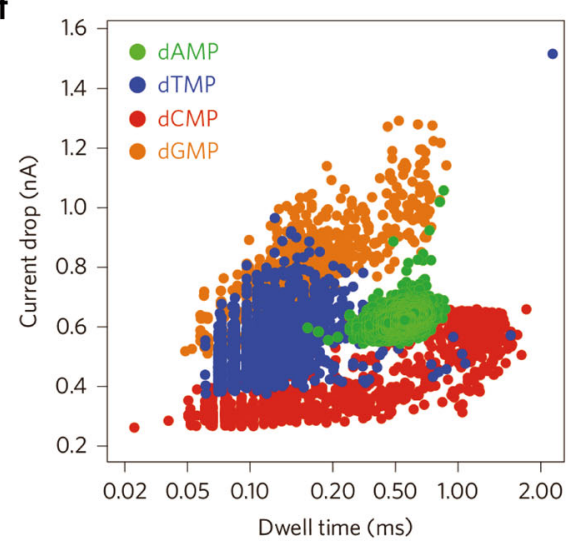

Fig. 8 Nanopore sequencing by ionic current. a Single-molecule DNA sequencing by ionic current measurements using MspA nanopores. The motor enzyme was attached to the pore, which enabled ratchet motions of single-stranded DNA via polymerase chain reactions. $\mathbf{b}$, $\mathbf{c}$ lonic conductance trace showing staircase-like profiles representing the distinct difference in the ionic current blockade by the nucleotides (b) that allowed one to determine the base sequence (c). Reproduced with permission from Noakes et al. ${ }^{87}$. d Single-molecule DNA detection using a MoS 2 nanopore. Polynucleotides were dispersed in an ionic liquid to slow the electrophoretic translocation speed. e Resistive pulses observed for 30-baselong oligonucleotides. $\mathbf{f}$ Single-nucleotide discriminations by resistive pulse height and width. Reproduced with permission from Feng et al. ${ }^{90}$.

single-molecule sensing with single-nucleobase resolution, despite not yet having been sequenced (Fig. $8 \mathrm{~d}-\mathrm{f})^{90}$. In contrast to this bottom-up approach, there are top-down methods to create ultrathin membranes. For instance, $\mathrm{SiN}_{\mathrm{x}}$ membranes can be locally thinned to $<5 \mathrm{~nm}$ thickness by dry etching, which enables excellent spatial resolution to discriminate the shapes of microRNAs by ionic current measurements ${ }^{92}$.

Progress in solid-state nanopore technologies has spurred many studies on sequencing proteins via nanopores ${ }^{93}$. It has been observed that the number of ionic current fluctuations matched that of residues in a protein during the electric field-driven movement of denatured protein through a subnanometer-diameter silicon nitride nanopore (Fig. 9a) ${ }^{93}$. Moreover, from the statistics of current fluctuation amplitudes, quadromers, molecules composed of four residues, can be discriminated by their primary structure ${ }^{93}$. These results suggest that these solid-state nanopores with subnanometer diameters are sufficiently sensitive to achieve a molecular volume difference space resolution as small as $0.07 \mathrm{~nm}^{3}$; thus, these nanopores are able to recognize the posttranslational modifications of single residues (Fig. 9b).
The compatibility of solid-state nanopores with NEMS technologies has also inspired researchers to incorporate additional probes to attain better discriminability of single molecules. Most strategies are based on embedding nanoelectrodes in a nanopore. For example, a pair of electrodes can be used to monitor transverse electron transport through single nucleotides upon DNA translocation (Fig. 10a). Since charge transport occurs by quantum tunneling through molecular orbitals, it allows us to distinguish the four types of nucleotides by their distinct electronic structures ${ }^{85,86,94,95}$. Indeed, molecular dynamics simulations suggest that by exploiting the difference between the highest occupied molecular orbital (HOMO) and lowest unoccupied molecular orbital (LUMO) of the four nucleotides, the transverse currents will be statistically discriminable for adenine, guanine, cytosine, and thymine (Fig. 10b $)^{94}$. It has been verified experimentally that the measured tunneling currents between ultrasharp electrodes are statistically distinguishable when different kinds of single nucleotides pass through the tunneling gap via Brownian motion in water (Fig. 10c ${ }^{86,96}$. The singlemolecule discriminability is further improved by 
a

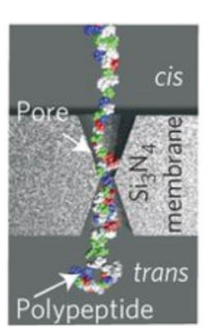

b

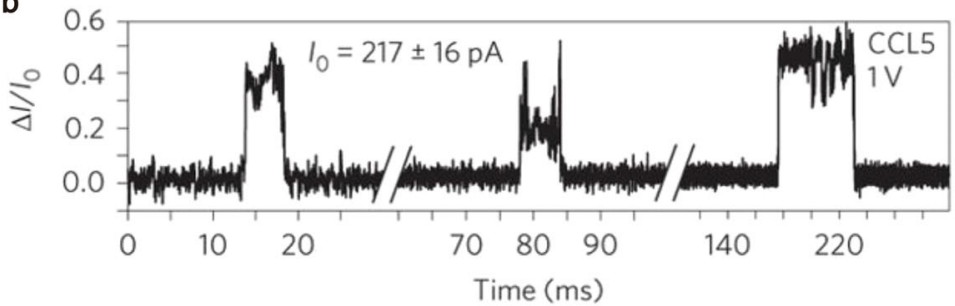

C

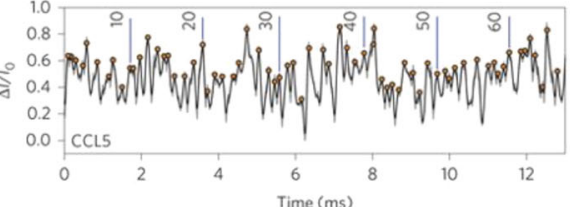

d

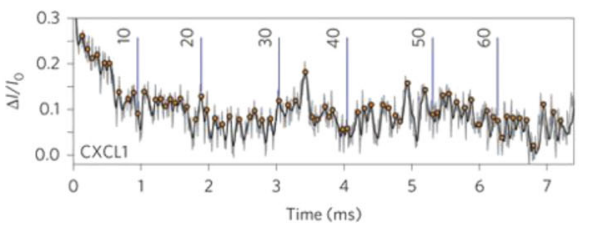

e

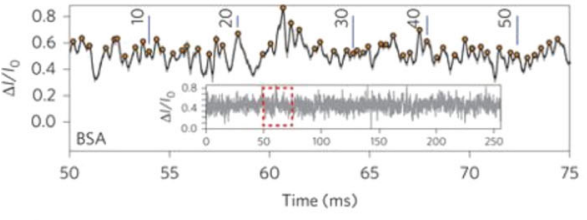

f

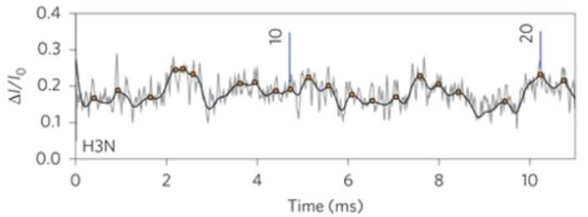

g

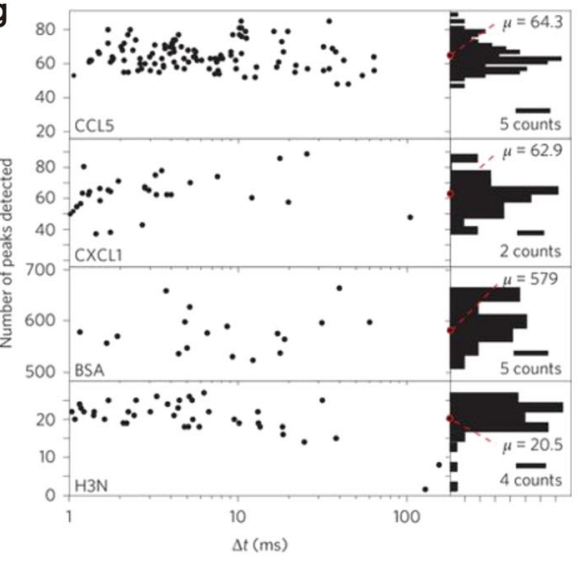

Fig. 9 Nanopore sequencing of polypeptides. a Schematic view of an electric field-driven polypeptide moving through a subnanometer-diameter $\mathrm{SiN}_{x}$ nanopore. $\mathbf{b}$ Monitored time-variant ionic current during translocations of single molecules of CCL5 through a $0.5 \times 0.6 \mathrm{~nm}^{2}$ pore at a $1-\mathrm{V}$ cross-pore voltage. c-f Expanded view of a single blockade associated with the translocation motion of single CCL5 (c), CXCL1 (d), BSA (e), and H3N (f) molecules. g Accounts of fluctuations tallied using a Fourier analysis from blockades shown in c-f. Reproduced with permission from Kennedy et al. ${ }^{93}$.

functionalizing electrode tips with recognition molecules so that the system becomes selective to particular types of nucleotides ${ }^{95,97}$. This method has also proven capable of identifying 12 different amino acids as well as a posttranslational modification ${ }^{98,99}$. After proof-of-principle demonstrations, various structures of electrode-embedded nanopores have been fabricated for sequencing by tunneling current. For instance, in-plane nanopores that make use of an electromigration break junction method to simultaneously form a nucleotide-sized electrode gap and nanopore (Fig. 10d ${ }^{100}$, metal-oxide-metal multilayer structures ${ }^{101}$, and cross-plane architectures using FIB milling/deposition ${ }^{102}$ or electron beam lithography (Fig. 10e) have been fabricated ${ }^{103}$. More recently, a tunneling current detector has been integrated at the tip of a nanopipette ${ }^{104}$, whose movable nature is expected to greatly expand the applications of the single-molecule sensor (Fig. 10f).
In addition to tunneling current methods, the field-effect transistor approach has been rigorously studied ${ }^{105-107}$, which can benefit from the larger current to sense small molecules ${ }^{108}$.

Despite the significant advances made in the past decade, several prominent challenges remain for the envisaged sensing of single-molecule sequencing by solid-state nanopores. Among the current issues, including the lack of affordable fabrication technologies for the mass production of single nanometer-sized pores ${ }^{40,42,109}$, a major concern has been that the translocation dynamics for detecting and discriminating single nucleotides ${ }^{110}$ is too fast, thus requiring an efficient method to regulate the electrophoretic motions of flexible biomolecules. More specifically, it is estimated that each pass-by nucleotide has to dwell within the nanoscopic sensing zone for at least $1 \mathrm{~ms}$ to provide useable current data ${ }^{1}$, while the actual 


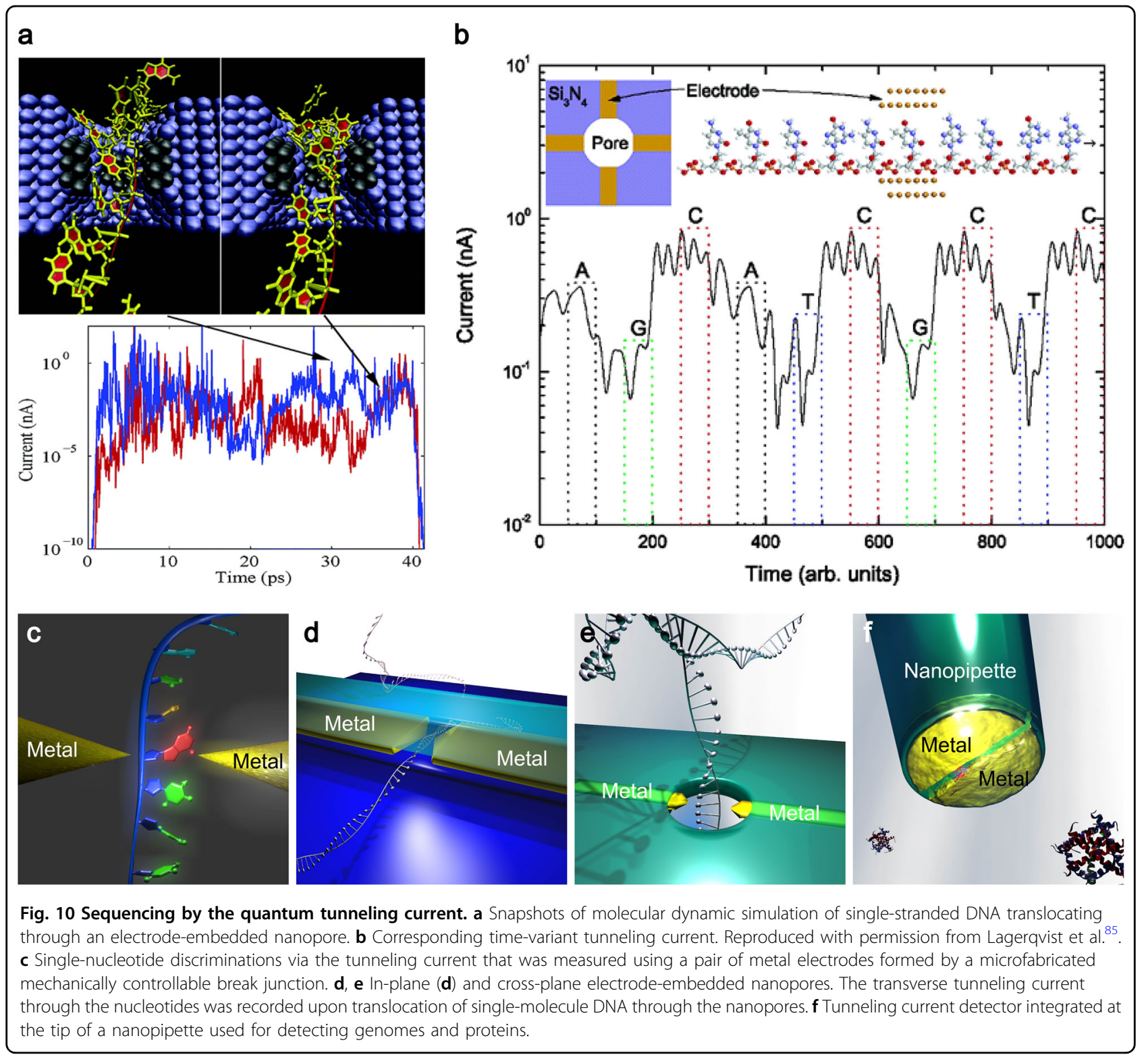

speed is $>1$ nucleotide per $\mu$ s when no means of translocation dynamics control is involved ${ }^{61,64}$. Several passive ways have been found to be useful to lower the translocation speed, such as lowering the temperature ${ }^{111}$, increasing the viscosity ${ }^{111,112}$, and imposing salt gradients $^{113}$. In particular, ionic liquids have been utilized in recent experiments as viscous media, helping to significantly slow the threading of DNA through a nanopore $^{90}$. Moreover, a means for active controls of rapid single-molecule motions has also been investigated by adding external stimuli, such as light irradiation ${ }^{114,115}$ and electric voltage application ${ }^{64,116}$. The latter approach is particularly promising because electrical manipulation can potentially implement fast feedback control against the instantaneous translocation dynamics of polynucleotides.
In fact, surround-gate electrode-embedded nanopores have been recently observed to provide fine control over the motions of nanoparticles in a nanopore at the $10 \mathrm{~nm} / \mathrm{s}$ level via gate voltage ${ }^{117}$. Although not yet applied to molecules such as proteins and genomes, electrical gating is expected to enable ultraslow translocation for solid-state nanopore sequencing.

\section{Plasmonic nanopore sensors}

While nanopore sensing is, in general, based on electrical measurements, there have also been developments to incorporate optical analyses. This technique uses plasmonic nanopores because they implement the high sensitivity of localized surface plasmon resonance (LSPR), which is a well-established technique exploited in various 
fields, such as photocatalysis and nanomedicine ${ }^{118,119}$. The sensor is composed of a pore in a solid membrane with plasmonic nanostructures ${ }^{120,121}$. It is known that the temporal resolution of the ionic current readout in ordinary nanopore sensors is severely restricted by the short dwell time of the molecules through the sensing region ${ }^{122}$. However, by introducing plasmonic sensing, the signal-to-noise ratio is enhanced through the confinement of the electromagnetic field at hotspots, thus improving the molecular detection capability. In addition, steep gradients in optical fields can be used to trap molecules in the sensing region, providing a long measurement time. One proposed device structure of a plasmonic nanopore is to fabricate plasmonic nanostructures next to nanopores. The advantage of this method lies in the nanopore being used to deliver the target molecules to the plasmonic hotspot through electrophoretic force, while the plasmonic nanostructure is used to provide the optical trapping force that helps to extend the molecule dwell time in the sensing region $^{123}$. Significant improvements in the signal-tobackground ratio, temporal resolution, sensor specificity, and scalable parallelized detection have been reported. Various types of plasmonic nanopore configurations, such as gold bowtie nanoantennas, bullseye-shaped plasmonic structures, plasmonic pore-in-cavities and graphene nanopores with self-integrated/aligned optical nanoantennas, have been developed ${ }^{124-127}$.

Recently, several novel techniques, such as plasmonic nanotrapping, fluorescence enhancement, and label-free optical sensing, including resonance shift and surfaceenhanced Raman spectroscopy (SERS), have been demonstrated. Protein detection and electrical biasing of plasmonic nanopores have been shown in a sensor consisting of an inverted-bowtie shaped opening in a gold-covered silicon-nitride membrane ${ }^{128,129}$. The nanoaperture serves both as an optical antenna and a through hole, allowing the simultaneous application of optical and electrokinetic forces, as shown in Fig. 11a. Protein beta-amylase has been detected by this device, where the polarity of the detected signal is predicted from the simulated plasmon resonance peak wavelength of the nanostructure (Fig. 11b).

Fluorescence emission can significantly enhance the signals within a plasmonic hotspot. Recently, a plasmonic nanowell-nanopore (PNW-NP) was developed, as shown in Fig. $11 \mathrm{c}^{129}$. Double-stranded DNA molecules of $\sim 5 \mathrm{kbp}$ labeled with a fluorophore were then detected by electrooptical measurements (Fig. 11d). A nearly tenfold increase in the peak intensities of the events was demonstrated with the PNW- NP device compared to that with a standard device, thereby showing the power of incorporating plasmonics and nanopores for molecular sensing.

Plasmonic nanopores can also be used for sensing unlabeled molecules by analyzing the resonance shift and surface-enhanced Raman spectroscopy (SERS) caused by the target molecules (Fig. 11e-g). The label-free sensing of individual DNA molecules at a high turnover rate has been demonstrated by integrating a nanopore with a plasmonic nanoantenna (Fig. 11e) ${ }^{130}$. The target biomolecules are electrophoretically driven to hotspot locations through the route guidance of the nanopore causing molecule-dependent quantitative changes in the refractive index (Fig. 11f) that even enables the identification of the four nucleobases (Fig. 11g) ${ }^{131}$.

\section{Nanoreactors}

Solid-state nanopores can not only serve as electrical sensing zones for counting and identifying individual particles and molecules but also provide a lithographically defined nanoscale space for chemical reactions that cannot be expected to occur in bulk systems. This observation is envisioned by the fact that mass transport across the membranes can be controlled by electrophoretic voltage. For example, reactants with positive and negative zeta potentials can be drawn into a pore, thereby enabling enhanced reaction rates to unconventional synthetic chemistry under highly nonequilibrium conditions. We herein introduce several cutting-edge works reporting the use of solid-state nanopore systems for these so-called nanoreactors.

Chemistry is the science of atoms, molecules, and their reactions. We know that in flasks, numerous reactants are free to diffuse in solvents via the available thermal energy to induce chemical reactions at a certain efficiency under equilibrium; this mechanism is dictated by, thermodynamics. On the other hand, the phenomenon in a confined nanoscopic space becomes quite different from that in bulk. For instance, chemical reactions in cells and organelles are intrinsically designed to take place in a surprisingly efficient manner, where the membranes play important roles in regulating the inner conditions ${ }^{132}$. Furthermore, advances in nanotechnologies offer new opportunities to form and leverage artificially designed nanoconfinements, which are called nanoreactors, for synthesizing new molecules at a low energy cost ${ }^{133-135}$. Along with various nanostructures from three to zero dimensions that have been tested in the past, such as metal nanoparticles ${ }^{136}$, metal-organic frameworks ${ }^{137}$, mesoporous materials ${ }^{138}$, and even virions ${ }^{139}$, solid-state nanopores have also proven useful to physically control space-limited chemical reactions, as we describe below.

Lin et al. ${ }^{140}$ used a track-etched nanopore with a double-conical shape and demonstrated a local change in $\mathrm{pH}$ in the confined space via glucose oxidase, and this change was yielded by the enzymatically activated sequential reactions between glucose and horseradish peroxidase; thus, they observed $\mathrm{pH}$-gated ion transport characteristics through ionic current measurements. 


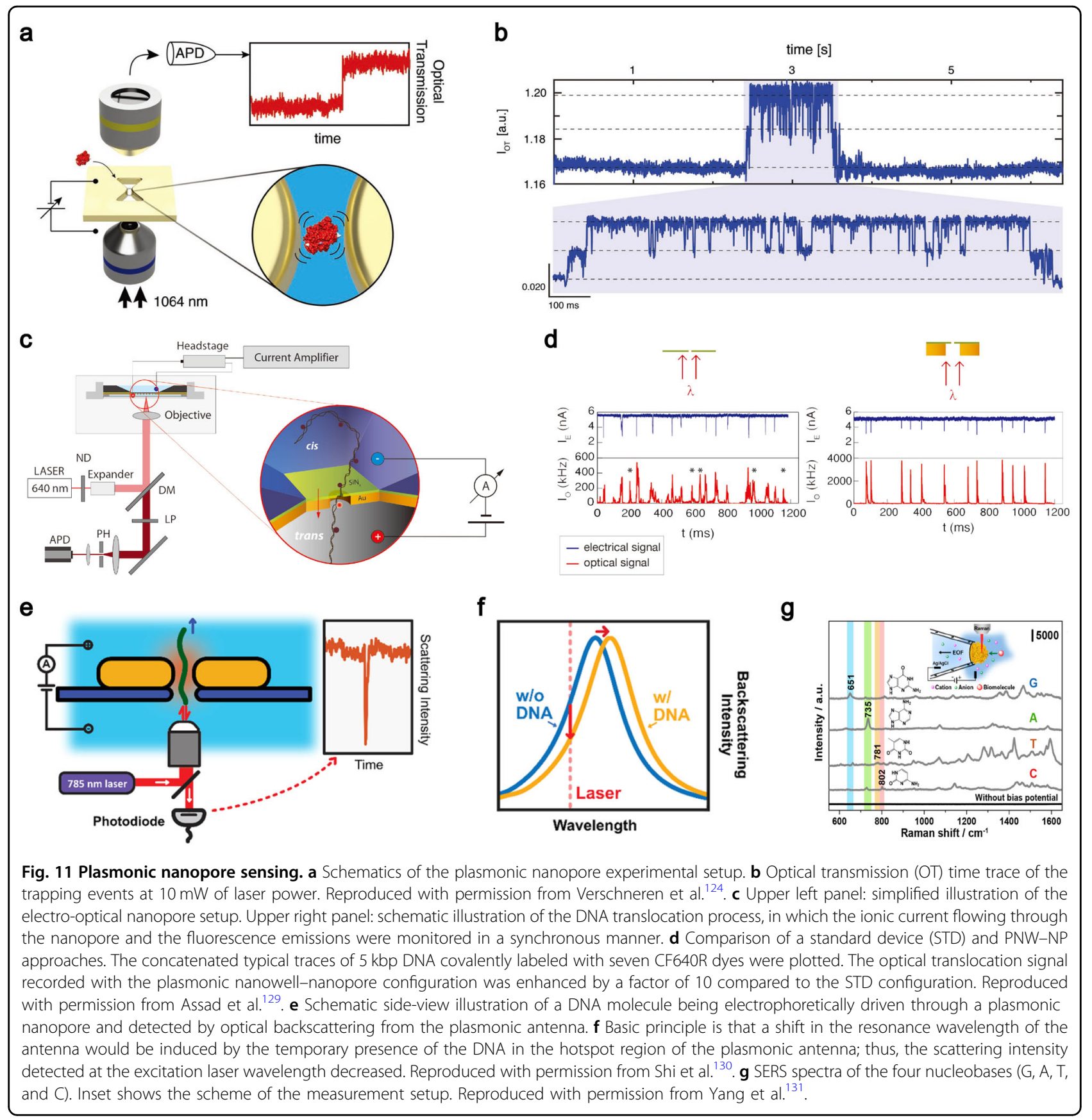

The concept of nanochannel reactors has been further applied to exploit gas-solid-liquid interfaces. Mi et al. ${ }^{141}$ utilized a porous anodic alumina membrane as a platform to create a confined nanospace for activating enzymes. For this, the nanopores were functionalized to immobilize glucose oxidase on one side of the orifices. By further tailoring the hydrophobicity of the channels, the enzyme molecules were made to touch both the liquid and gas media. The formed gas-solid-liquid interfaces enabled an 80-fold enhancement in the catalytic efficiency of glucose oxidase, which was ascribed to the facilitated access of the gas phase oxygen molecules to the reaction field.

Nanofabricated channels have also proven to be applicable as a template for synthesizing nanomaterials. Venta et al. ${ }^{142}$ employed electron-beam-sculpted several nanometer-sized $\mathrm{SiN}_{\mathrm{x}}$ nanopores as a nanoreactor. They applied an electric voltage across the membrane to move both the cationic (hydrazine) and anionic (gold(III) chlorides) reactants toward the nanopore. Electrically controlled molecular transport led to enhanced frequency 
for these molecules to come across with each other for reactions that enabled an orders of magnitude faster synthesis of Au nanoparticles. Later, Wood and Zhang ${ }^{143}$ applied the approach for synthesizing a gold nanowire in a $\mathrm{SiO}_{2}$ nanopore template. These innovative findings provide a way to fabricate zero- and one-dimensional nanostructures with well-controlled sizes at predefined positions.

\section{Potential applications: nanoporous iontronic devices}

Decades of progress in nanopore science and technologies have led to the development of novel nanofluidic channel structures along with the discovery of numerous ion transport phenomena. Currently, the abundance of ionic current characteristics of solid-state nanopores have been increasingly studied in their own right from the viewpoints of not only academic interest but also for practical applications beyond sensors, such as iontronic building blocks for nanopore diodes, energy harvesters, and even neuromorphic devices.

\section{Nanopore diodes and transistors}

Iontronics is an emerging idea for making use of the ion transport properties of fluidic channels as analogs of solidstate electronic components. This field originates from our knowledge of the fascinating functions of brains programmed to transduce electrical signals in aqueous circuits of neural networks via ion transport, thereby implementing various biologically vital functions. Due to their nanoscale dimensions, nanopore systems offer distinct ionic current behaviors that cannot be obtained in their bulk counterparts. In this section, we highlight one of the most fundamental yet practically important properties of ionic current rectifications in solid-state nanopores.

A diode is one of the basic elements in electrical circuits aiming at restricting the electrical current in one direction. By utilizing the ICR effect discussed previously, nanofluidic versions of diodes have been realized ${ }^{144,145}$. A schematic view of one structure is illustrated in Fig. $12 \mathrm{a}^{145}$ : the channel surface of a nanopore is chemically modified to be asymmetric along the axial direction of the channel, where one half is patterned with cationic molecules while the other half is patterned with another type of electrically neutral molecule. The left half of this channel allows only $\mathrm{Cl}^{-}$to pass through, while the right half admits both $\mathrm{K}^{+}$ and $\mathrm{Cl}^{-}$(Fig. 12b). Therefore, a forward bias with an electric field pointing from the neutral half to the positive half will cause ion accumulation near the interface between the left and right halves of the channel, while a reverse bias will lead to a concentration depletion near the interface (Fig. 12c, d) ${ }^{145}$. As a result, a large electrical current occurs only in one direction, akin to the rectification characteristics of diodes. The experimentally measured current-voltage characteristics obtained by using $10 \mathrm{mM} \mathrm{KCl}$ in nanopores with a length of $120 \mu \mathrm{m}$, a width of $4 \mu \mathrm{m}$, and a height of $\sim 30 \mathrm{~nm}$ are plotted with solid circles in Fig. 12e, $\mathrm{f}^{145}$. The results are well explained by the numerical simulation (solid lines in the figure) based on a simplified 1D electrokinetic model.

After intensive studies on the physics behind asymmetric ion transport, more efforts have been devoted to achieving higher rectification ratios. For example, 2D nanopores in bilayered hexagonal boron nitride membranes have been proposed to be an excellent rectifier ${ }^{146}$. In this study, two channel motifs with triangular and hexagonal shapes are used for molecular dynamics simulations (Fig. 12g). Owing to the bipolar membrane surface charge properties of the former structure, diodelike behavior is observed with a high rectification ratio of 50 for the latter structure with a size of $0.75 \mathrm{~nm}$ (Fig. 12h). The importance of the surface charge asymmetry was also suggested in the theoretical work by Ma et al. ${ }^{147}$, wherein the rectification ratio was revealed to change by two orders of magnitude depending on the surface charge profiles inside and outside the nanopores (Fig. 12i). Further experimental investigation is needed to realize practical nanopore diodes based on these innovative device concepts.

In addition to diodes, solid-state nanopores can be used as transistors. By adding a gate electrode, the electrical potential and ion concentration inside the channel can be adjusted (Fig. 13a), similar to the working principle of a field-effect transistor $(\text { FET })^{148}$. Here, the crucial issue of successful gate manipulation is the electrical tuning of the effective surface charge density $\sigma^{*}$ on the channel wall: $\sigma^{\prime \prime}=\sigma_{\mathrm{W}}+\varepsilon_{\mathrm{D}} E_{\mathrm{D}}$, where $\sigma_{\mathrm{W}}$ is the original density of the surface charge on the wall, $\varepsilon_{\mathrm{D}}$ is the permittivity of the gate dielectric, and $E_{\mathrm{D}}$ is the gate voltage-induced transverse electric field at the channel surface. By using electron-beam lithography and reactive ion etching (RIE) processes, nanopores with diameters as small as $10 \mathrm{~nm}$ and surrounded by $\mathrm{SiO}_{2}$ gate dielectric films that are $\sim 40 \mathrm{~nm}$ thick have been fabricated. Such a thin gate dielectric enhances $E_{\mathrm{D}}$, thereby enabling the tuning of surface charge through gate voltage. The transfer curves are then measured as seen in Fig. $13 b^{148}$. The experimental results indicate that the smaller the imposed $\mathrm{KCl}$ concentration is, the larger the amplitude of the gate tuned ionic current. In contrast, there is no obvious change in the current when the concentration is $>0.1 \mathrm{M}$. This result is just as expected since the ion concentration within the channel is determined by the wall surface charge density when using a low salt concentration. Thus, the associated channel conductance shows a dependence on the gate voltage through the tuned surface charge density of the wall. The demonstrated properties of ionic FETs may find promising applications in the field of 


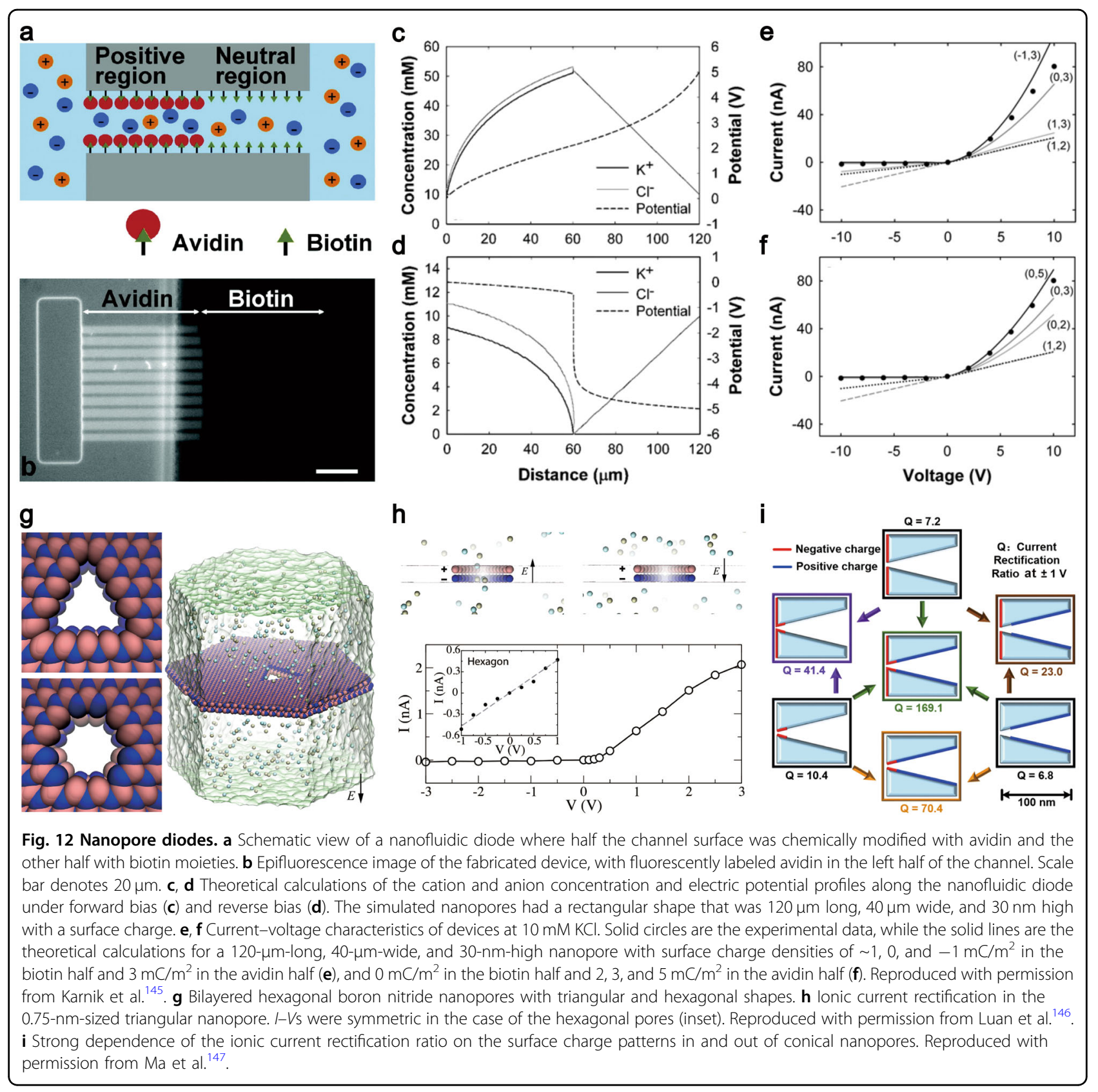

nanofluidic-integrated circuits and the manipulation of ions and biomolecules.

More recently, nanopore technologies have allowed the formation of ionic bipolar junctions for electrical gating of cross-membrane ionic currents ${ }^{149}$. These junctions consist of a pore drilled in a membrane composed of a $\mathrm{SiN}_{\mathrm{x}} /$ Nafion/ $\mathrm{SiN}_{\mathrm{x}}$ multilayer (Fig. 13c). The $\mathrm{SiN}_{\mathrm{x}}$ surfaces are functionalized to possess a positive charge so that the pore regions at the top and bottom are anion rich. In contrast, the Nafion film serves as a source of cations, thereby comprising an ionic npn junction. This novel design enables gate control to amplify the ionic current via the gate voltage added to the electrode embedded in the copolymer. Biomimicking three-terminal circuits is expected to pave the way for establishing experimental models of neuronal signaling.

\section{Energy harvesters}

Unlike charge transport in solid matter, fluid flow has nonnegligible impacts on charge transport in nanopores. This in turn enables energy harvesters that convert mechanical energy into electricity.

Generally, there are two major approaches of energy harvesting using nanopore devices: pressure-driven and 

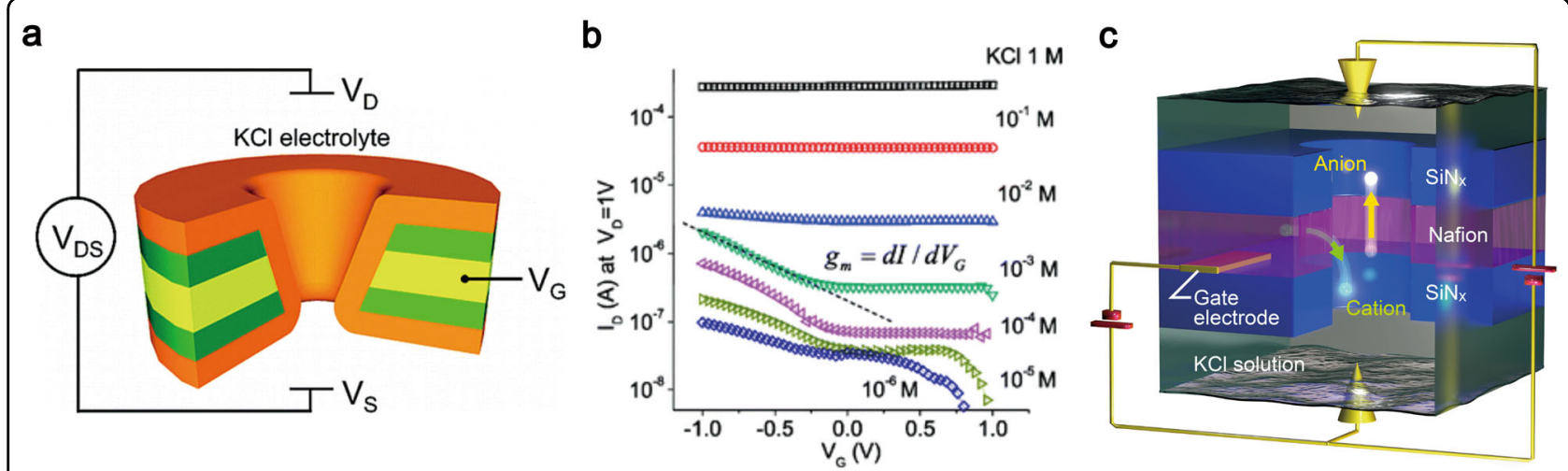

Fig. 13 Nanopore transistors. a lon concentration profile and transport were manipulated by the surrounding gate dielectric $\left(\mathrm{TiO}_{2}\right.$, orange) and electrode (TiN, yellow). $\mathbf{b}$ Gate modulation of ionic current at various $\mathrm{KCl}$ concentrations. Reproduced with permission from Vlassiouk et al. ${ }^{144}$. c Three-terminal nanopore system forming an ionic npn junction.

sea/river salt concentration bias-driven approaches. Regarding the former, the basic principle of converting mechanical energy into electrical energy through a nanopore system is demonstrated in Fig. 6b, where the pressure-driven transport of counterions within the EDL gives rise to the electrical potential/current. By utilizing the pressure-driven streaming current, electrical power generation in nanopores with a height of $75 \mathrm{~nm}$ is demonstrated to reach a conversion efficiency of $\sim 3 \%{ }^{150}$. It is further suggested that to achieve optimal energy conversion efficiency, nanopores with a small crosssection and slippery channel walls should be used along with a dilute salt concentration ${ }^{77,151}$. Other strategies have also been proposed to further enhance the energy conversion efficiency, such as exploiting large ions so that high concentrations can be produced far from the walls to utilize the large flow velocity in that area ${ }^{152}$.

On the other hand, it has long been known through thermodynamics that Gibbs free energy will be released when solutions with different salt concentrations are mixed. Quantitatively, nearly $2 \times 10^{12}$ of electric power may be harvestable during this process, given the huge amount of river water $\left(37,000 \mathrm{~km}^{3}\right)$ streaming into the sea globally. Such an alluring prospect of clean and sustainable energy has triggered a tremendous amount of research on developing power converting devices that are highly efficient, have a low cost and are able to be mass produced. As discussed previously, RED in nanopores can be used to harness the thermal energy within the salt gradient. Experimentally, silica, alumina, polyimide, boron nitride nanotubes, and so on have been utilized, and electrical output power stimulated by a saline concentration bias has been reported ${ }^{5,65,66,79,80,153,154}$. The power generation per unit channel volume is $\sim 7.7 \mathrm{~W} / \mathrm{m}^{2}$ in silica nanopores ${ }^{61}$. Recently, Feng et al. ${ }^{78}$ demonstrated the use of $2 \mathrm{D}$ molybdenum disulfide $\left(\mathrm{MoS}_{2}\right)$ nanopore systems as nanoscale power generators, and an estimated power density of up to $10^{6} \mathrm{~W} / \mathrm{m}^{2}$ was extracted from a $\mathrm{KCl}$ salt gradient. Theoretically, the relation between the output power of nanofluidic devices and their parameters has been analyzed based on an electrokinetic model that offers a novel approach of the multisectional use of the sea/river salt bias for gaining improved energy conversion efficiency $^{82}$.

\section{Desalination}

Perhaps the most straightforward applications of solidstate nanopores are filters. In particular, nanopore systems are considered a promising structure for the desalination of sea water.

The shortage of clean water is one major problem that restricts many developing countries/regions from improving the health of their people, building modern agriculture or developing water-consuming industries. However, the mainstream desalination process currently used, namely, reverse osmosis, requires a considerable amount of energy: reverse osmosis consumes $3.5-5.0 \mathrm{kWh}$ of energy and emits $1.6 \mathrm{~kg}$ of $\mathrm{CO}$ to produce $1 \mathrm{~m}^{3}$ of freshwater ${ }^{155}$. With the advances of 2D nanopore techniques, an energyefficient and cost-saving method to desalinate sea water for a sustainable fresh water supply has emerged. It was first proposed theoretically that nanometer-sized pores in single-layer graphene would be able to prevent salt-ion passage while allowing water molecules to permeate through $^{156}$. More precisely, a salt rejection rate of nearly $100 \%$ and rapid water transport as large as $10^{6} \mathrm{~g} \mathrm{~m}^{-2} \mathrm{~s}^{-1}$ at $40^{\circ} \mathrm{C}$ has been achieved by experiments using graphene nanopores ${ }^{157}$. Furthermore, a similar efficiency of $88 \%$ ion rejection has been reported by simulating the desalination process in single-layer molybdenum disulfide $\left(\mathrm{MoS}_{2}\right)$ nanopores ${ }^{158}$. Experimental and theoretical studies on this topic have suggested that there are quite a few unique advantages when utilizing graphene or other 2D nanopore systems for desalination ${ }^{159}$. First, technically, it has the 


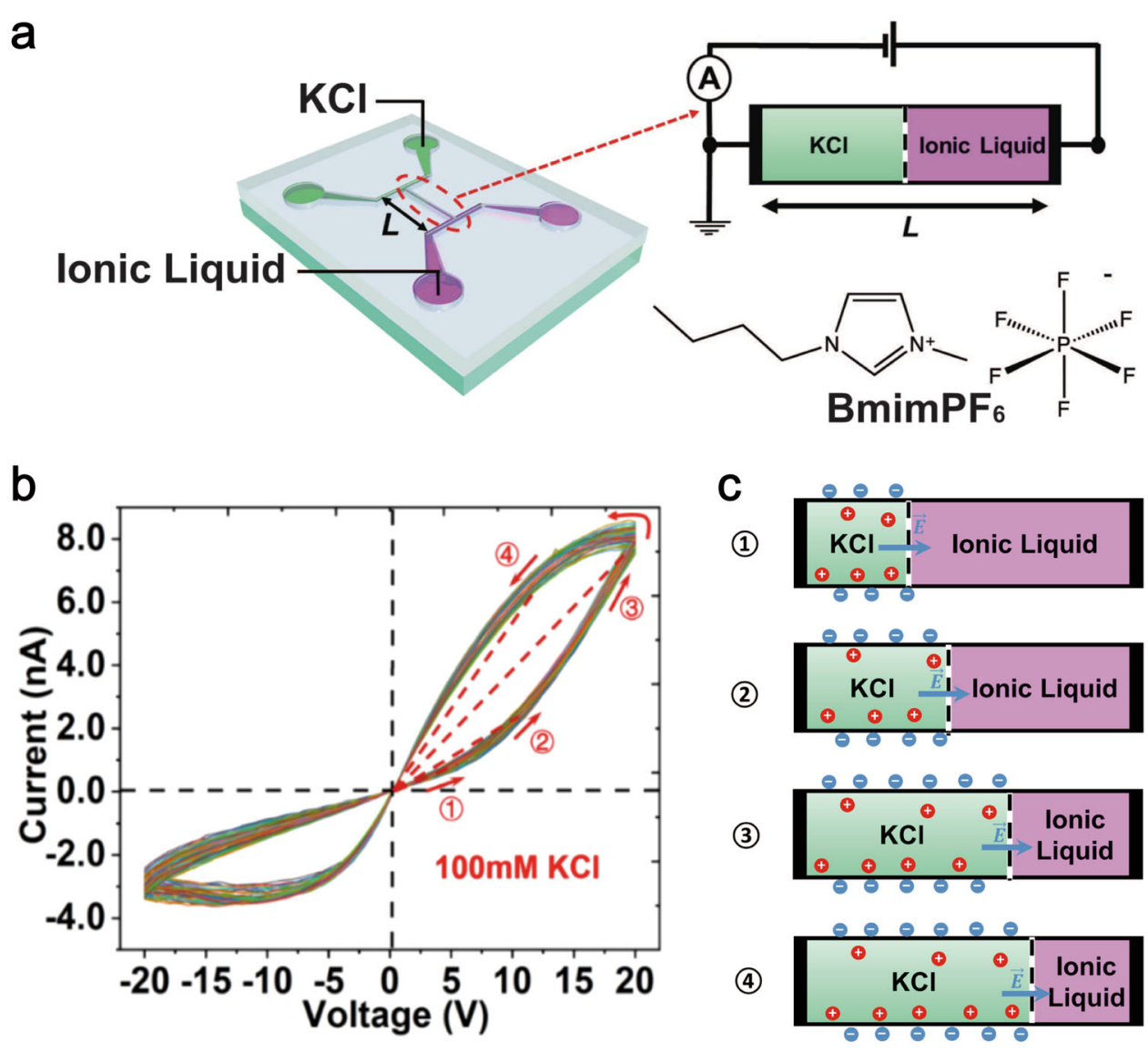

d
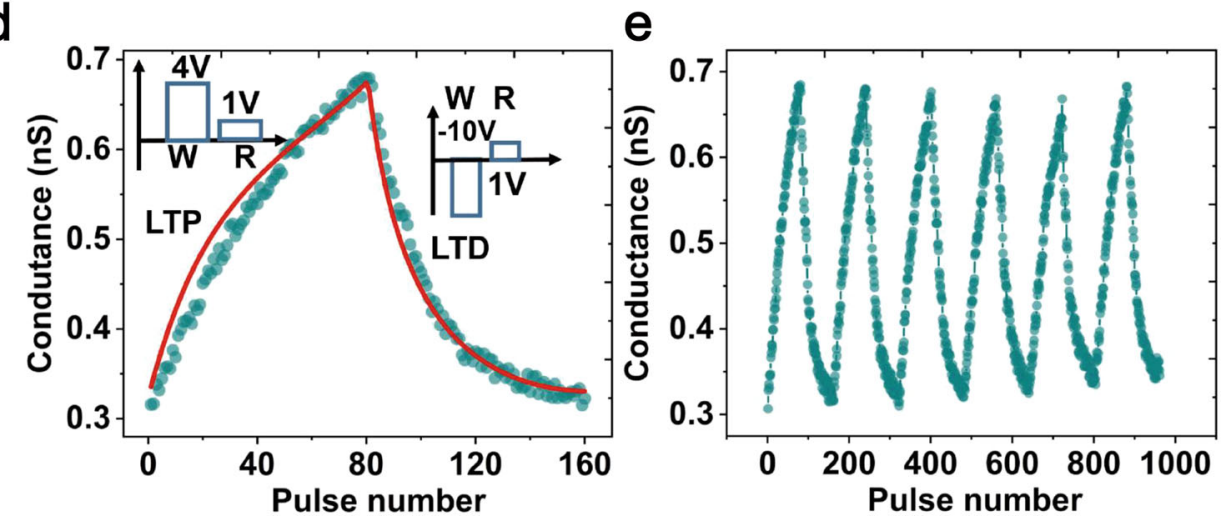

Fig. 14 Nanopore memristor. a $\mathrm{KCl}$ solution and room-temperature ionic liquid ( $\mathrm{BmimPF}_{6}$ ) were separately added to the two ends of a nanopore, and an interface formed between the two types of immiscible liquid. b Measured $/(V)$ sweeping curve where the $100-\mathrm{mM} \mathrm{KCl}_{\text {solution }}$ and $\mathrm{BmimPF}_{6}$ IL were separately added to the two ends of a nanopore. $\mathbf{c}$ The numbers in circles indicate several subsequent points during voltage sweeping, while the slopes of the dashed red lines in the figure indicate the corresponding conductance. Right: corresponding physical pictures of the points shown in the left figure. Measured conductance tuning of nanopore synapses $\mathbf{d}$ under a series of SET pulses followed by another series of RESET pulses and e under several cycles of SET and RESET pulse trains. Reproduced with permission from refs. ${ }^{167}$.

potential to massively produce nanopores with just the right dimension of filtering water molecules on 2D membranes, which will greatly enhance the desalination efficiency. Second, rather than a slow diffusion process of reverse osmosis, nanopore desalination is basically a sieving method. By optimizing the nanopore parameters, such as the pore size and chemical functionality of the surface, fast water transport, and desalination can be achieved. Last but not least, 2D materials are in general sufficiently robust owing to the strong covalent bonds between intralayer atoms; thus, 2D nanopores demonstrate stable performance in harsh practical situations, 
such as fouling and mechanical pressure, during the desalination process.

\section{Nanopore memristors and neuromorphic devices}

Massively parallel implementations of biological synapses realize various vital tasks of organisms. Solid-state nanopores have also been demonstrated to act as memristors and emulate synapse functionalities, which is a fundamental step toward neuromorphic computing. This research is expected to open a novel horizon of nanofluidic devices. Since it is rather foreign outside of nanofluid society, we present a brief introduction of memristors as well as challenges and perspectives of this new topic.

A memristor was proposed by Leon Chua in the 1970s when he noticed that there was no electrical element characterizing the relation between the physical quantities of electrical charge $q$ and magnetic flux $\Phi^{160}$. The original memristor $M$ was then mathematically defined as:

$$
M=\frac{\mathrm{d} \Phi}{\mathrm{d} q}
$$

Note that the unit of the memristor $M$ is the same as that of the resistor $R$. However, the electrical resistance of the memristor here is no longer a constant. Instead, it depends on the history of electrical current flowing through the device. Quantitatively, the present resistance of the memristor is determined by the amount of net charge that is transported through the device. Moreover, the resistance modification is preserved even after the electrical stimulus is turned off. In this aspect, the device keeps memory of its electrical history and hence is named a "memristor".

In 2008, a long-missing memristor was claimed to be found in a $\mathrm{Pt} / \mathrm{TiO}_{2} / \mathrm{Pt}$ device by a team in $\mathrm{HP} \mathrm{Labs}^{161}$ based on the observed nonvolatile and continuous tuning of conductance. Since its first experimental demonstration, memristors have attracted considerable interest from researchers in the past decade, and nonvolatile conductance tuning mechanisms for various kinds of materials have been utilized to realize memristors ${ }^{162,163}$. The thriving of memristor-related research is largely ascribed to its promise as a hardware building block for nextgeneration in-memory computing schemes such as neuromorphic computing. ${ }^{164,165}$ Neuromorphic computing was a revolutionary concept proposed by Carver Mead in the 1980s as computing in similar ways as biological brain computing ${ }^{166}$. At the hardware level, it calls for individual devices that can emulate the functions of synapses and neurons to accelerate the neuromorphic computing speed while optimizing the power consumption efficiency. In this respect, memristors are regarded as one of the most promising candidates to implement synaptic functions since nonvolatile conductance tuning can be used directly to mimic changes in synaptic strength. Ideally, the synaptic conductance should increase linearly from $G_{\text {min }}$ to $G_{\max }$ given a series of SET pulse trains while decreasing vice versa in the presence of another series of RESET pulses. The corresponding physical mechanism for this ideal behavior is that the interface between regions with high (undoped) and low resistivity (doped) is shifted forward/backward in a gradual manner under a SET/RESET pulse train. However, in real memristive synapses, such strictly linear and highly reproducible conductance tuning behaviors are seldom reported owing to the intrinsic randomness of the conductance tuning processes within widely studied solid-state memristive materials.

The above discussions further indicate that it is the search, design and fabrication of interfacial memristors that lie at the heart of achieving ideal synaptic functions. To address this prominent challenge, nanopore-based memristors have been proposed and experimentally demonstrated $^{167}$. One design capable of realizing gradual and long-term conductance tuning is shown in Fig. 14. A nanopore tens of micrometers long, hundreds of nanometers wide and tens of nanometers high connecting two microchambers is fabricated. $\mathrm{A} \mathrm{KCl}$ solution and roomtemperature ionic liquid (IL) are then injected into the two ends of the nanopore. An interface emerges between the two kinds of immiscible liquids, as shown schematically in the inset of Fig. 14a. Since the conductivity gap between the $\mathrm{KCl}$ solution and $\mathrm{IL}$ can be several orders of magnitude when using $100 \mathrm{mM} \mathrm{KCl}$, the $\mathrm{KCl} / \mathrm{IL}$ nanopore system now serves as an interfacial-type memristor with a clearly defined high/low resistance border. Moreover, the interface can be driven back-and-forth by imposing a sweeping voltage on the nanopore owing to the effect of electroosmotic flow. The experimentally measured cycles of currentvoltage sweeping $I(V)$ using $100 \mathrm{mM} \mathrm{KCl}$ and a 1-butyl-3methylimidazolium hexafluorophosphate $\left(\mathrm{BmimPF}_{6}\right) \mathrm{IL}$ in a $10-\mu \mathrm{m}$ long, 200-nm wide, and 63-nm high rectangular nanopore are shown in Fig. 14b, while the physical pictures for several points during $I(V)$ sweeping are sketched in Fig. 14c.

The measured analog weight modulation of nanoporebased synapses, which is defined as the conductance change under a series of SET voltage pulses followed by another series of RESET pulses, is shown in Fig. 14d, e. Excellent linearity, symmetry and $\mathrm{C} 2 \mathrm{C}$ consistency are observed, indicating the great promise of nanopore devices as synapses ${ }^{167}$. As analyzed previously, here, the property of an interfacial-type memristor plays a crucial role in presenting highly linear, symmetrical and reproducible conductance tuning behaviors.

The above discussion outlines that the use of a highly viscous IL is crucial to realize a nanopore-based interfacial-type memristor, not only because of its immiscibility with a $\mathrm{KCl}$ solution but also because of its large 
viscosity, which helps stabilize the displaced interface between $\mathrm{KCl} / \mathrm{IL}$ to realize long-term conductance tuning. However, a side effect is that the operational speed of this memristor is quite slow, since the viscous IL severely slows the electroosmotic flow. The currently demonstrated write speed of nanopore-based synapses is at the level of seconds per operation ${ }^{167}$, while those of solidstate RRAM are approximately nanoseconds ${ }^{168}$. Further strategies to lower the operation speed into the range for practical use are thereby in urgent demand. Several approaches are suggested here ranging from device geometry optimization to material design. One is to keep scaling down the nanopore dimensions so that the wall surface effect is further enhanced, thereby increasing the strength of the driving force. Another is to explore more kinds of ionic liquids with substantially smaller viscosities while still being immiscible with a $\mathrm{KCl}$ solution.

Today, the most popular architecture of artificial synaptic arrays that connects the presynaptic layer of neurons to postsynaptic arrays is the crossbar, which has been realized in several works. The top electrodes are connected to the bottom electrodes via holes at the crosspoints, and memristive materials are filled in the holes as synapses. Accordingly, the crossbar structure of the synaptic matrix calls for the mass fabrication of 3D nanopore arrays and associated diversion channels when applied to nanofluidic-based neural networks.

Thanks to the biocompatibility of nanopore devices with biological systems, another potential application yet to explore is their use as brain-machine interface (BMI) devices. Currently, BMI systems are usually composed of arrays of microelectrodes with which the electrical signals of the cerebral cortex are measured. The recorded signals are then transferred to the in vitro information processing system and analyzed. However, since nanopore devices share quite similar work media, such as $\mathrm{K}^{+}$ and $\mathrm{Cl}^{+}$, with biological nerve cells, it may be possible to connect nanopore-based neuromorphic devices directly to neurons. Hence, the information may be transferred bidirectionally in a much more efficient way between biological nerve systems and nanopore-based neuromorphic chips, while also being processed more intelligently by the latter.

\section{Conclusions and future perspectives}

In conclusion, we have presented a panoramic view of nanopore-based transport, from the materials and techniques employed to fabricate nanopores to the novel transport phenomena within these channels and the underlying mechanisms along with promising applications in various fields. From the discussion presented above, it becomes clear that the surface properties of nanopore walls dominate the ion and liquid flow within the channels, and by exploiting and modulating these properties, revolutionary application scenarios are created. One central topic is to understand, manipulate and then utilize a charged solution within a channel induced by the surface charge on the nanopore wall. Through electrical means, electroosmotic flow will be stimulated and regulated, which may be useful in controlling biomolecule translocation motion through the nanopore toward the goal of electrical identification of single molecules. In addition, mechanical or Gibbs thermal energy can be converted into electrical power through the hydrodynamic pressure- or salt concentration biasgenerated ionic current in nanopores.

Novel materials, particularly 2D materials as membranes for drilling nanopores, have opened new horizons in nanofluidic research. The ultralow thickness-todiameter aspect ratio of graphene nanopores has created a biomolecule sensing zone with subnanometer spatial resolution, which outperforms biological nanopore sensors. Another unique advantage of $2 \mathrm{D}$ materialbased nanopore systems is the ultrahigh structural strengths of the materials, even in the presence of densely drilled nanometer-sized holes. Therefore, these materials may be quite promising for application in water desalination since 2D nanopores are able to block the transport of ions while allowing water molecules to still pass through.

Novel device designs have also given rise to fairly new transport phenomena and associated applications. One intriguing configuration of nanofluidic devices is to separately fill the channel with two types of immiscible liquids from each end and then impose electrical tuning. Memristive behaviors have been demonstrated by manipulating the electroosmotic flow inside. The unique advantage of nanofluidic-based memristors lies in that they are intrinsically interfacial memristors: there exists a clear interface between the high- and low-resistance regions formed by the two immiscible liquids with different resistivities, and this interface is shifted gradually by the imposed electrical voltage. An interfacial memristor has long been desired by important applications, such as memristor-based artificial synapses (memristive synapses) in neuromorphic computing, because this type of memristor cannot be realized based on conventional solid-state resistive switching materials. The corner of the curtain of this new and exciting application scenario has been raised and now needs more research attention to innovate the future.

\footnotetext{
Acknowledgements

Y.H. thanks the support from the National Natural Science Foundation of China under No. 61974051. M.T. acknowledges support from the Japan Society for the Promotion of Science (JSPS) KAKENHI grant numbers $18 \mathrm{H01846}$ and $19 \mathrm{H} 02574$.
}

Conflict of interest

The authors declare no competing interests. 


\section{Publisher's note}

Springer Nature remains neutral with regard to jurisdictional claims in published maps and institutional affiliations.

Received: 17 November 2020 Revised: 13 March 2021 Accepted: 14 April 2021.

Published online: 11 June 2021

\section{References}

1. Branton, D. et al. The potential and challenges of nanopore sequencing. Nat. Biotechnol. 26, 1146-1153 (2008).

2. Venkatesan, B. M. \& Bashir, R. Nanopore sensors for nucleic acid analysis. Nat. Nanotechnol. 6, 615 (2011).

3. Dekker, C. Solid-state nanopores. Nat. Nanotechnol. 2, 209-215 (2007).

4. Derrington, I. M. et al. Nanopore DNA sequencing with MspA. Proc. Natl Acad. Sci. USA 107, 16060 (2010).

5. Zhang, Y., Huang, Z., He, Y. \& Miao, X. Enhancing the efficiency of energy harvesting from salt gradient with ion-selective nanochannel. Nanotechnology 30, 295402 (2019).

6. Bocquet, L. \& Tabeling, P. Physics and technologiccal aspects of nanofluidics. Lab Chip 14, 3143-3158 (2014).

7. Haywood, D. G., Saha-Shah, A., Baker, L. A. \& Jacobson, S. C. Fundamental studies of nanofluidics: nanopores, nanochannels, and nanopipets. Anal. Chem. 87, 172-187 (2015).

8. Xu, Y. Nanofluidics: a new arena for materials science. Adv. Mater. 30, 1702419 (2018).

9. Nanofluidics in on the rise. Nat. Mater. 19, 253 (2020).

10. Bocquet, L. Nanofluidics coming of age. Nat. Mater. 19, 254-256 (2020).

11. Li, J. et al. lon-beam sculpting at nanometre length scales. Nature 412, 166 (2001).

12. Venta, K. et al. Differentiation of short, single-stranded DNA homopolymers in solid-state nanopores. ACS Nano 7, 4629-4636 (2013).

13. van den Hout, M. et al. Controlling nanopore size, shape and stability. Nanotechnology 21, 115304 (2010).

14. Masuda, H. \& Fukuda, K. Ordered metal nanohole arrays made by a two-step replication of honeycomb structures of anodic alumina. Science $\mathbf{2 6 8}, 1466$ (1995).

15. Tabrizi, M. A., Ferre-Borrull, J. \& Marsal, L. F. Remote biosensor for the determination of trypsin by using nanoporous anodic alumina as a threedimensional nanostructured material. Sci. Rep. 10, 2356 (2020).

16. Jessensky, O., Müller, F. \& Gösele, U. Self-organized formation of hexagonal pore arrays in anodic alumina. Appl. Phys. Lett. 72, 1173-1175 (1998).

17. Keller, F., Hunter, M. S. \& Robinson, D. L. Structural features of oxide coatings on aluminum. J. Electrochem. Soc. 100, 411-419 (1953).

18. Diggle, J. W., Downie, T. C. \& Goulding, C. W. Anodic oxide films on aluminum. Chem. Rev. 69, 365-405 (1969).

19. Masuda, H. et al. Highly ordered nanochannel-array architecture in anodic alumina. Appl. Phys. Lett. 71, 2770-2772 (1997).

20. Yuan, J. H., He, F. Y., Sun, D. C. \& Xia, X. H. A simple method for preparation of through-hole porous anodic alumina membrane. Chem. Mater. 16, 1841-1844 (2004).

21. Harrell, C. C., Siwy, Z. S. \& Martin, C. R. Conical nanopore membranes: controlling the nanopore shape. Small 2, 194-198 (2006).

22. Xie, Y. et al. Electric energy generation in single track-etched nanopores. Appl. Phys. Lett. 93, 163116 (2008).

23. Wang, L. et al. A method to tune the ionic current rectification of tracketched nanopores by using surfactant. Phys. Chem. Chem. Phys. 13, 576-581 (2011).

24. Kwok, H., Briggs, K. \& Tabard-Cossa, V. Nanopore fabrication by controlled dielectric breakdown. PLOS ONE 9, e92880 (2014).

25. Yanagi, I., Akahori, R., Hatano, T. \& Takeda, K.-I. Fabricating nanopores with diameters of sub-1 $\mathrm{nm}$ to $3 \mathrm{~nm}$ using multilevel pulse-voltage injection. Sci. Rep. 4, 5000 (2014).

26. Briggs, $K$. et al. Kinetics of nanopore fabrication during controlled breakdown of dielectric membranes in solution. Nanotechnology 26, 084004 (2015).

27. Yanagi, l., Akahori, R. \& Takeda, K-I. Stable fabrication of a large nanopore by controlled dielectric breakdown in a high-pH solution for the detection of various-sized molecules. Sci. Rep. 9, 13143 (2019).
28. Zhang, Y. et al. Solid state nanopores: nanopore formation via tip-controlled local breakdown using an atomic force microscope. Small Methods $\mathbf{3}$ 1970018 (2019).

29. Vogel, R. et al. Quantitative sizing of nano/microparticles with a tunable elastomeric pore sensor. Anal. Chem. 83, 3499-3506 (2011).

30. Willmott, G. R. et al. Use of tunable nanopore blockade rates to investigate colloidal dispersions. J. Phys. Condens. Matter. 22, 454116 (2010).

31. Sha, J., Ni, Z., Liu, L., Yi, H. \& Chen, Y. A novel method of fabricating a nanopore based on a glass tube for single-molecule detection. Nanotechnology 22, 175304 (2011).

32. Karhanek, M., Kemp, J. T., Pourmand, N., Davis, R. W. \& Webb, C. D. Single DNA molecule detection using nanopipettes and nanoparticles. Nano Lett. $\mathbf{5}$ 403-407 (2005).

33. Umehara, S., Karhanek, M., Davis, R. W. \& Pourmand, N. Label-free biosensing with functionalized nanopipette probes. Proc. Natl Acad. Sci. USA 106 4611-4616 (2009).

34. Douglas, S. M. et al. Rapid prototyping of 3D DNA-origami shapes with caDNAno. Nucleic Acids Res. 37, 5001-5006 (2009).

35. Hernández-Ainsa, S. \& Keyser, U. F. DNA origami nanopores: developments, challenges and perspectives. Nanoscale 6, 14121-14132 (2014).

36. Bell, N. A. W. et al. DNA origami nanopores. Nano Lett. 12, 512-517 (2012).

37. Hernández-Ainsa, S. et al. DNA origami nanopores for controlling DNA translocation. ACS Nano 7, 6024-6030 (2013).

38. Burns, J. R., Stulz, E. \& Howorka, S. Self-assembled DNA nanopores that span lipid bilayers. Nano Lett. 13, 2351-2356 (2013).

39. Siwy, Z. S. \& Davenport, M. Graphene opens up to DNA. Nat. Nanotechnol. $\mathbf{5}$ 697-698 (2010).

40. Schneider, G. F. et al. DNA translocation through graphene nanopores. Nano Lett. 10, 3163-3167 (2010).

41. Garaj, S. et al. Graphene as a subnanometre trans-electrode membrane. Nature 467, 190-193 (2010).

42. Merchant, C. A. et al. DNA translocation through graphene nanopores. Nano Lett. 10, 2915-2921 (2010).

43. Zhou, Z. et al. DNA translocation through hydrophilic nanopore in hexagonal boron nitride. Sci. Rep. 3, 3287 (2013).

44. Liu, K. Feng, J., Kis, A. \& Radenovic, A. Atomically thin molybdenum disulfide nanopores with high sensitivity for DNA translocation. ACS Nano 8 2504-2511 (2014).

45. Mojtabavi, M., VahidMohammadi, A., Liang, W., Beidaghi, M. \& Wanunu, M. Single-molecule sensing using nanopores in two-dimensional transition metal carbide (MXene) membranes. ACS Nano 13, 3042-3053 (2019).

46. Siwy, Z. S. \& Howorka, S. Engineered voltage-responsive nanopores. Chem. Soc. Rev. 39, 1115-1132 (2010).

47. Siwy, Z. et al. Preparation of synthetic nanopores with transport properties analogous to biological channels. Surf. Sci. 532-535, 1061-1066 (2003).

48. Ali, M., Ramirez, P., Mafé, S., Neumann, R. \& Ensinger, W. A pH-tunable nanofluidic diode with a broad range of rectifying properties. ACS Nano $\mathbf{3}$, 603-608 (2009).

49. Yameen, B. et al. lonic transport through single solid-state nanopores controlled with thermally nanoactuated macromolecular gates. Small $\mathbf{5}$ 1287-1291 (2009).

50. Iqbal, S. M., Akin, D. \& Bashir, R. Solid-state nanopore channels with DNA selectivity. Nat. Nanotechnol. 2, 243 (2007)

51. Yusko, E. C. et al. Controlling protein translocation through nanopores with bio-inspired fluid walls. Nat. Nanotechnol. 6, 253-260 (2011).

52. Krishnakumar, P. et al. Slowing DNA translocation through a nanopore using a functionalized electrode. ACS Nano 7, 10319-10326 (2013).

53. Hou, X., Guo, W. \& Jiang, L. Biomimetic smart nanopores and nanochannels. Chem. Soc. Rev. 40, 2385-2401 (2011).

54. Hall, J. E. Access resistance of a small circular pore. J. Gen. Physiol. 66, 531 (1975).

55. Lee, C. et al. Large apparent electric size of solid-state nanopores due to spatially extended surface conduction. Nano Lett. 12, 4037-4044 (2012).

56. Sahu, S. \& Zwolak, M. Maxwell-Hall access resistance in graphene nanopores, Phys. Chem. Chem. Phys. 20, 4646 (2018).

57. Kowalczyk, S. W., Grosberg, A. Y., Rabin, Y. \& Dekker, C. Modeling the conductance and DNA blockade of solid-state nanopores. Nanotechnology 22, 315101 (2011).

58. Heerema, S. J. et al. 1/f noise in graphene nanopores. Nanotechnology $\mathbf{2 6}$, 074001 (2015) 
59. Hyun, C., Rollings, R. \& Li, J. L. Probing access resistance of solid-state nanopores with a scanning-probe microscope tip. Small 8, 385-392 (2012).

60. Stein, D., Kruithof, M. \& Dekker, C. Surface-charge-governed ion transport in nanofluidic channels. Phys. Rev. Lett. 93, 035901 (2004).

61. Smeets, R. M. M. et al. Salt dependence of ion transport and DNA translocation through solid-state nanopores. Nano Lett. 6, 89-95 (2006).

62. Yan, Y., Wang, L., Xue, J. \& Chang, H.-C. lon current rectification inversion in conic nanopores: nonequilibrium ion transport biased by ion selectivity and spatial asymmetry. J. Chem. Phys. 138, 044706 (2013).

63. van der Heyden, F. H. J., Stein, D. \& Dekker, C. Streaming currents in a single nanofluidic channel. Phys. Rev. Lett. 95, 116104 (2005).

64. He, Y., Tsutsui, M., Fan, C., Taniguchi, M. \& Kawai, T. Controlling DNA translocation through gate modulation of nanopore wall surface charges. ACS Nano 5, 5509-5518 (2011).

65. Siria, A. et al. Giant osmotic energy conversion measured in a single transmembrane boron nitride nanotube. Nature 494, 455-458 (2013).

66. Kim, D. K., Duan, C. H., Chen, Y. F. \& Majumdar, A. Power generation from concentration gradient by reverse electrodialysis in ion-selective nanochannels. Microfluid. Nanofluid. 9, 1215-1224 (2010).

67. Kim, S. J., Wang, Y. C., Lee, J. H., Jang, H. \& Han, J. Concentration polarization and nonlinear electrokinetic flow near a nanofluidic channel. Phys. Rev. Lett. 99, 044501 (2007).

68. Siwy, Z. S. Ion-current rectification in nanopores and nanotubes with broken symmetry. Adv. Funct. Mater. 16, 735-746 (2006).

69. Huang, $X$., Kong, X. - Y., Wen, L. \& Jiang, L. Bioinspired ionic diodes: From unipolar to bipolar. Adv. Funct. Mater. 28, 1801079 (2018).

70. Lin, C. Y., Wong, P. H., Wang, P. H., Simy, Z. S. \& Yeh, L. H. Electrodiffusioosmosis-induced negative differential resistance in phregulated mesopores containing purely monovalent solutions. ACS Appl. Mater. Interfaces 12, 3198-3204 (2020).

71. Leong, I. W. et al. Quasi-stable salt gradient and resistive switching in solidstate nanopores. ACS Appl. Mat. Interfaces 12, 52175-52181 (2020).

72. Lin, K., Lin, C. -Y., Polster, J. W., Chen, Y. \& Siwy, Z. S. Charge inversion and calcium gating in mixtures of ions in nanopores. J. Am. Chem. Soc. 142, 2925-2934 (2020).

73. Haywood, D. G., Harms, Z. D. \& Jacobson, S. C. Electroosmotic flow in nanofluidic channels. Anal. Chem. 86, 11174-11180 (2014).

74. Peng, R. \& Li, D. Electroosmotic flow in single PDMS nanochannels. Nanoscale 8, 12237-12246 (2016).

75. Karmi, A. et al. Detection of Au nanoparticles using peptide-modified $\mathrm{Si}_{3} \mathrm{~N}_{4}$ nanopores. ACS Appl. Nano Mater. 4, 1000-1008 (2021).

76. Chau, C. C., Ralford, S. E., Hewitt, E. W. \& Actis, P. Macromolecular crowding enhances the detection of DNA and proteins by a solid-state nanopore. Nano Lett. 20, 5553-5561 (2020).

77. van der Heyden, F. H. J., Bonthuis, D. J., Stein, D., Meyer, C. \& Dekker, C. Electrokinetic energy conversion efficiency in nanofluidic channels. Nano Lett. 6, 2232-2237 (2006).

78. Feng, J. et al. Single-layer $\mathrm{MoS}_{2}$ nanopores as nanopower generators. Nature 536, 197-200 (2016)

79. Guo, W. et al. Energy harvesting with single-ion-selective nanopores: A concentration-gradient-driven nanofluidic power source. Adv. Funct. Mater. 20, 1339-1344 (2010).

80. Kim, J., Kim, S. J. \& Kim, D. K. Energy harvesting from salinity gradient by reverse electrodialysis With anodic alumina nanopores. Energy 51, 413-421 (2013).

81. Yang, M. et al. Anomalous effects of water flow through charged nanochannel membranes. RSC Adv. 4, 26729-26737 (2014).

82. He, Y. et al. Electrokinetic analysis of energy harvest from natural salt gradients in nanochannels. Sci. Rep. 7, 13156 (2017).

83. Kasianowicz, J. J., Brandin, E., Branton, D. \& Deamer, D. W. Characterization of individual polynucleotide molecules using a membrane channel. Proc. Nat Acad. Sci. USA 93, 13770 (1996).

84. Fologea, D. et al. Detecting single stranded DNA with a solid state nanopore Nano Lett. 5, 1905-1909 (2005).

85. Lagerqvist, J., Zwolak, M. \& Di Ventra, M. Fast DNA sequencing via transverse electronic transport. Nano Lett. 6, 779-782 (2006)

86. Tsutsui, M., Taniguchi, M., Yokota, K. \& Kawai, T. Identifying single nucleotides by tunnelling current. Nat. Nanotechnol. 5, 286 (2010).

87. Noakes, M. T. et al. Increasing the accuracy of nanopore DNA sequencing using a time-varying cross membrane voltage. Nat. Biotechnol. 37, 651-656 (2019).
88. Tyler, A. D. et al. Evaluation of Oxford nanopore's MinlON sequencing device for microbial whole genome sequencing applications. Sci. Rep. 8, 10931 (2018)

89. Liu, S. et al. Boron nitride nanopores: highly sensitive DNA single-molecule detectors. Adv. Mater. 25, 4549-4554 (2013).

90. Feng, J. et al. Identification of single nucleotides in $\mathrm{MoS}_{2}$ nanopores. Nat. Nanotechnol. 10, 1070 (2015).

91. Schneider, G. F. et al. Tailoring the hydrophobicity of graphene nanopore for its use as nanopores for DNA translocation. Nat. Commun. 4, 2619 (2013).

92. Wanunu, M. et al. Rapid electronic detection of probe-specific microRNAs using thin nanopore sensors. Nat. Nanotechnol. 5, 807-814 (2010).

93. Kennedy, E., Dong, Z., Tennant, C. \& Timp, G. Reading the primary structure of a protein with $0.07 \mathrm{~nm}^{3}$ resolution using a subnanometre-diameter pore. Nat. Nanotechnol. 11, 968 (2016).

94. Zwolak, M. \& Di Ventra, M. Electronic signature of DNA nucleotides via transverse transport. Nano Lett. 5, 421-424 (2005).

95. Huang, S. et al. Identifying single bases in a DNA oligomer with electron tunnelling. Nat. Nanotechnol. 5, 868 (2010).

96. Chang, $\mathrm{S}$. et al. Electronic signatures of all four DNA nucleosides in a tunneling gap. Nano Lett. 10, 1070-1075 (2010).

97. He, H. et al. Functionalized nanopore-embedded electrodes for rapid DNA sequencing. J. Phys. Chem. C 112, 3456-3459 (2008).

98. Ohshiro, T. et al. Detection of post-translational modifications in single peptides using electron tunnelling currents. Nat. Nanotechnol. 9, 835 (2014).

99. Di Muccio, G., Rossini, A. E., Di Marino, D., Zollo, G. \& Chinappi, M. Insights into protein sequencing with an a-Hemolysin nanopore by atomistic simulations. Sci. Rep. 9, 6440 (2019).

100. Tsutsui, M. et al. Single-molecule sensing electrode embedded in-plane nanopore. Sci. Rep. 1, 46 (2011).

101. Pang, P. et al. Fixed-gap tunnel junction for reading DNA nucleeotides. ACS Nano 8, 11994-12003 (2014).

102. Ivanov, A. P. et al. DNA tunneling detector embedded in a nanopore. Nano Lett. 11, 279-285 (2011).

103. Fanget, A. et al. Nanopore integrated nanogaps for DNA detection. Nano Lett. 14, 244-249 (2014).

104. Tang, L. et al. Combined quantum tunnelling and dielectrophoretic trapping for molecular analysis at ultra-low analyte concentrations. Nat. Commun. 12, 913 (2021).

105. Xie, P., Xiong, Q., Fang, Y., Qing, Q. \& Lieber, C. M. Local electrical potential detection of DNA by nanowire-nanopore sensors. Nat. Nanotechnol. 7 119-125 (2012).

106. Graf, M., Lihter, M., Altus, D. A., Marion, S. \& Radenovic, A. Transverse detection of DNA using a MoS , nanopore. Nano Lett. 19, 9075-9083 (2019).

107. Parkin, W. M. \& Drndic, M. Signal and noise in FET-nanopore devices. ACS Sens. 3, 313-319 (2018)

108. Saha, K. K., Drndic, M. \& Nikolic, B. K. DNA base-soecific modulation of microampere transverse edge currents through a metallic graphene nanoribbon with a nanopore. Nano Lett. 12, 50-55 (2012).

109. He, Y. et al. Graphene/hexagonal boron nitride/graphene nanopore for electrical detection of single molecules. npg Asia Mater. 6, e104 (2014).

110. He, Y., Tsutsui, M., Fan, C., Taniguchi, M. \& Kawai, T. Gate manipulation of DNA capture into nanopores. ACS Nano 5, 8391-8397 (2011).

111. Fologea, D., Uplinger, J., Thomas, B., McNabb, D. S. \& Li, J. Slowing DNA translocation in a solid-state nanopore. Nano Lett. 5, 1734-1737 (2005).

112. Plesa, C., van Loo, N. \& Dekker, C. DNA nanopore translocation in glutamate solutions. Nanoscale 7, 13605-13609 (2015).

113. Wanunu, M., Morrison, W., Rabin, Y., Grosberg, A. Y. \& Meller, A. Electrostatic focusing of unlabelled DNA into nanoscale pores using a salt gradient. Nat. Nanotechnol. 5, 160-165 (2010).

114. Keyser, U. F. et al. Direct force measurements on DNA in a solid-state nanopore. Nat. Phys. 2, 473-477 (2006).

115. Fiori, N. D. et al. Optoelectronic control of surface charge and translocation dynamics in solid-state nanopores. Nat. Nanotechnol. 8, 946-951 (2013).

116. Freedman, K. J. et al. Nanopore sensing at ultra-low concentrations using single-molecule dielectrophoretic trapping. Nat. Commun. 7, 10217 (2016).

117. Tsutsui, M. et al. Field effect control of translocation dynamics in surroundgate nanopores. Commun. Mater. 2, 29 (2021).

118. Zeng, S., Baillargeat, D., Ho, H.P. \& Yong, K.-T. Nanomaterials enhanced surface plasmon resonance for biological and chemical sensing applications. Chem. Soc. Rev. 43, 3426-3452 (2014). 
119. Zhao, H., Brown, P. H. \& Schuck, P. On the distribution of protein refractive index increments. Biophys. J. 100, 2309-2317 (2011).

120. Spitzberg, J. D., Zrehen, A., van Kooten, X. F. \& Meller, A. Plasmonic-nanopore biosensors for superior single-molecule detection. Adv. Mater. 31, 1900422 (2019).

121. Garoli, D., Yamazaki, H., Maccaferri, N. \& Wanunu, M. Plasmonic nanopores for single-molecule detection and manipulation: Toward sequencing applications. Nano Lett. 19, 7553-7562 (2019).

122. Plesa, $\mathrm{C}$. et al. Fast translocation of proteins through solid state nanopores. Nano Lett. 13, 658-663 (2013).

123. Belkin, M., Chao, S.-H., Jonsson, M. P., Dekker, C. \& Aksimentiev, A. Plasmonic nanopores for trapping, controlling displacement, and sequencing of DNA ACS Nano 9, 10598-10611 (2015).

124. Verschueren, D., Shi, X. \& Dekker, C. Nano-optical tweezing of single proteins in plasmonic nanopores. Small Methods 3, 1800465 (2019).

125. Crick, C. R. et al. Low-noise plasmonic nanopore biosensors for single molecule detection at elevated temperatures. ACS Photon. 4, 2835-2842 (2017).

126. Nicoli, F., Verschueren, D., Klein, M., Dekker, C. \& Jonsson, M. P. DNA translocations through solid-state plasmonic nanopores. Nano Lett. 14, 6917-6925 (2014).

127. Li, Y. et al. Photoresistance switching of plasmonic nanopores. Nano Lett. 15 776-782 (2015).

128. Chen, $C$. et al. Enhanced optical trapping and arrangement of nano-objects in a plasmonic nanocavity. Nano Lett. 12, 125-132 (2012).

129. Assad, O. N. et al. Light-enhancing plasmonic-nanopore biosensor for superior single-molecule detection. Adv. Mater. 29, 1605442 (2017).

130. Shi, X., Verschueren, D. V. \& Dekker, C. Active delivery of single DNA molecules into a plasmonic nanopore for label-free optical sensing. Nano Lett. 18, 8003-8010 (2018).

131. Yang, J.-M. et al. Surface-enhanced raman scattering probing the translocation of DNA and amino acid through plasmonic nanopores. Anal. Chem. 91 6275-6280 (2019).

132. van Roekel, H. W. H. et al. Programmable chemical reaction networks: emulating regulatory functions in living cells using a bottom-up approach. Chem. Soc. Rev. 44, 7465-7483 (2015).

133. Yu, Z., Zhou, P., Pan, W. \& Tang, B. A biomimetic nanoreactor for synergistic chemiexcited photodynamic therapy and starvation therapy against tumor metastasis. Nat. Commun. 9, 5044 (2018).

134. Lee, J., Kim, S. M. \& Lee, I. S. Functionalization of hollow nanoparticles for nanoreactor applications. Nano Today 9, 631-667 (2014).

135. Godoy-Gallardo, M., York-Duran, M. J. \& Hosta-Rigau, L. Recent progress in micro/nanoreactores toward the creation of artificial organelles. Adv. Health Mater. 7, 1700917 (2018).

136. Lee, J., Park, J. C. \& Song, H. A nanoreactor framework of a Au@SiO 2 yolk/shell structure for catalytic reduction of p-nitrophenol. Adv. Mater. 20, 1523-1528 (2008)

137. Lian, X. et al. Enzyme-MOF nanoreactor activates nontoxic paracetamol for cancer therapy. Angew. Chem. 130, 5827-5832 (2018).

138. Ding, S. et al. Formation of $\mathrm{SnO}_{2}$ hollow nanospheres inside mesoporous silica nanoreactors. J. Am. Chem. Soc. 133, 21-23 (2011).

139. Liu, A., Traulsen, C. H.-H. \& Cornelissen, J. L. M. Nitroarene reduction by a virus protein cage based nanoreactor. ACS Sens. 6, 3084-3091 (2016).

140. Lin, L., Yan, J. \& Li, J. Small-molecule triggered cascade enzymatic catalysis in hour-glass shaped nanochannel reactor for glucose monitoring. Anal. Chem. 86, 10546-10551 (2014).

141. Mi, L. et al. Boosting gas involved reactions at nanochannel reactor with joint gas-solid-liquid interfaces and controlled wettability. J. Am. Chem. Soc. 139 10441-10446 (2017).
142. Venta, K., Wanunu, M. \& Drndic, M. Electrically controlled nanoparticle synthesis inside nanopores. Nano Lett. 13, 423-429 (2013).

143. Wood, M. \& Zhang, B. Bipolar electrochemical method for dynamic in situ control of single metal nanowire growth. ACS Nano 9, 2454-2464 (2015).

144. Vlassiouk, I. \& Siwy, Z. S. Nanofluidic diode. Nano Lett. 7, 552-556 (2007).

145. Karnik, R., Duan, C., Castelino, K., Daiguji, H. \& Majumdar, A. Rectification of ionic current in a nanofluidic diode. Nano Lett. 7, 547-551 (2007).

146. Luan, B. \& Zhou, R. Atomic-scale fluidic diodes based on triangular nanopores in bilayer hexagonal boron nitride. Nano Lett. 19, 977-982 (2019).

147. Ma, L. et al. Modulation of ionic current rectification in ultrashort conical nanopores. Anal. Chem. 92, 16188-16196 (2020).

148. Nam, S.-W., Rooks, M. J., Kim, K.-B. \& Rossnagel, S. M. Ionic field effect transistors with sub-10 nm multiple nanopores. Nano Lett. 9, 2044-2048 (2009).

149. Lucas, R. A., Lin, C. -Y., Baker, L. A. \& Siwy, Z. S. lonic amplifying circuits inspired by electronics and biology. Nat. Commun. 11, 1568 (2020).

150. van der Heyden, F. H., Bonthuis, D. J., Stein, D., Meyer, C. \& Dekker, C. Power generation by pressure-driven transport of ions in nanofluidic channels. Nano Lett. 7, 1022-1025 (2007).

151. Chang, C. C. \& Yang, R. J. Electrokinetic energy conversion efficiency in ionselective nanopores. Appl. Phys. Lett. 99, 083102 (2011).

152. Gillespie, D. High energy conversion efficiency in nanofluidic channels. Nano Lett. 12, 1410-1416 (2012).

153. Chein, R. \& Liu, B. Energy conversion from electrolyte concentration gradient using charged nanopores. Int. J. Green Energy 13, 1400-1411 (2016).

154. Hwang, J., Kataoka, S., Endo, A. \& Daiguji, H. Enhanced energy harvesting by concentration gradient-driven ion transport in SBA-15 mesoporous silica thin films. Lab Chip 16, 3824-3832 (2016).

155. Elimelech, M. \& Phillip, W. A. The future of seawater desalination: energy, technology, and the environment. Science 333, 712 (2011).

156. Cohen-Tanugi, D. \& Grossman, J. C. Water desalination across nanoporous graphene. Nano Lett. 12, 3602-3608 (2012).

157. Heiranian, M., Farimani, A. B. \& Aluru, N. R. Water desalination with a singlelayer $\mathrm{MoS}_{2}$ nanopore. Nat. Commun. 6, 8616 (2015).

158. Surwade, S. P. et al. Water desalination using nanoporous single-layer graphene. Nat. Nanotechnol. 10,459-464 (2015).

159. Ramanathan, A. A., Aqra, M. W. \& Al-Rawajfeh, A. E. Recent advances in 2D nanopores for desalination. Environ. Chem. Lett. 16, 1217-1231 (2018).

160. Chua, L. Memristor-the missing circuit element. IEEE Trans. Circuit Theory 18, 507-519 (1971).

161. Strukov, D. B., Snider, G. S., Stewart, D. R. \& Williams, R. S. The missing memristor found. Nature 453, 80-83 (2008).

162. Hu, M. et al. Memristor-based analog computation and neural network classification with a dot product engine. Adv. Mater. 30, 1705914 (2018).

163. Wang, Z. et al. Fully memristive neural networks for pattern classification with unsupervised learning. Nat. Electron. 1, 137-145 (2018).

164. Jo, S. H. et al. Nanoscale memristor device as synapse in neuromorphic systems. Nano Lett. 10, 1297-1301 (2010).

165. Li, Y., Wang, Z., Midya, R., Xia, Q. \& Yang, J. J. Review of memristor devices in neuromorphic computing: materials sciences and device challenges. J. Phys. D Appl. Phys. 51, 503002 (2018).

166. Mead, C. Neuromorphic electronic systems. Proc. IEEE 78, 1629-1636 (1990).

167. Zhang, P. et al. Nanochannel-based transport in an interfacial memristor can emulate the analog weight modulation of synapses. Nano Lett. 19 4279-4286 (2019).

168. Yao, P. et al. Face classification using electronic synapses. Nat. Commun. 8 15199 (2017). 